\title{
EXOTISCHE WILDTIERE IM 1. JT. V. CHR. IM MEDITERRANEN RAUM UND IRLAND IM ARCHÄOLOGISCHEN BEFUND
}

Mirjam MAHN

THE ARCHAEOLOGICAL EVIDENCE OF EXOTIC WILD ANIMALS DURING THE $1^{\text {sT }}$ MILLENNIUM BC IN EUROPE

Since the term „exotic" is not recorded before the time of Plautus (um 254-184 v. Chr.) and in both latin and greek written sources of the Roman period never appears in connection with animals, it is used in this paper as a terminus technicus. Regarded as exotic will be animals, which by the means of human activity got introduced into regions not belonging to their range of endemic distribution. In antic premonetary european societies animals and their products represented proven values next to other goods. As hard to achieve, expensive rarities exotic animals are usually associated with consume, commerce orientated economy or with prestigious pet owning. In Greece remains of exotic wild animals are closely associated with religious contexts. A Barbary ape found in the settlement of Navan Fort, Northern Ireland, was certainly a valued pet. However the status of the fallow deer western of Greece during the $1^{\text {st }}$ millennium $\mathrm{BC}$ is unknown. It remains unclear, whether this species is endemic to southern Italy and Sicily, or got re-imported by Phoenicians and used as a tame resource of meat after a postulated extinction prior to the Bronze Age. Furthermore every picture of long-range trade would be incomplete without regarding the exports leaving Europe. However that is an archaeozoologically sparsely explored subject up to now. During the second half of the $1^{\text {st }}$ millennium BC coinage trade gradually substituted traditional exchange trade in the Mediterranean. The decreasing importance of the later most likely had its effect on commerce with animals and their raw material, but is difficult to prove. In Greece this alteration may display itself by a change of offering practice: Subsequent to the Archaic period coin donations superseded individual goods as common offerings. In trade with animals or animal products other economical purposes and ideal values turned more into focus. The customization to exotic valuables associated with luxury created demands, which Phoenicians knew to further develop and the Greeks and later on the Romans appreciated to cultivate. Maybe this tendency led together with other factors towards the since Hellenistic times increasing decadence especially of the Romans, to enjoy the value of rare foreign animals through the stomach or in circus displays and animal hunts.

Keywords: animal bones; animal products; exotic animals; wild animals; $1^{\text {st }}$ millennium $\mathrm{BC}$; trade; Europe; Archaeozoology

\section{WAS IST EXOTISCH?}

Etymologisch bezeichnet das griechische $\varepsilon \xi \dot{\omega} \tau$ เ $о \varsigma$, lat. exoticus das außerhalb Befindliche, Fremde. ${ }^{1}$ Die lateinische Bezeichnung ist erstmals im Euvre des Plautus (um 254-184 v. Chr.) nachgewiesen, im Griechischen ist sie sogar erst ab der römischen Kaiserzeit bei Porphyros (um 234-304 n. Chr.) ${ }^{2}$ belegt. Älter sind Formulierungen wie $\beta \alpha \rho \beta \alpha \rho o \varsigma$ und $\beta \alpha \rho \beta \alpha \rho o \varphi \omega ́ v \omega \varsigma(\beta \alpha \rho \beta \alpha \rho o \varphi \omega ́ v \omega \nu)^{3}$ oder

\footnotetext{
1 Zur philologischen Tradition des Begriffs Bodson 1998, 145-151.

${ }^{2}$ Als Substantiv in Porph. Abst. 4,6,6.

${ }^{3}$ Hom. Il. 2,867.
}

$\xi \varepsilon \tilde{v} o \varsigma^{4}$. Sie beziehen sich nach der Erkenntnis von L. Bodson allerdings auf die Herkunft von Menschen, nicht von Tieren - ebenso wenig wie das lateinische exoticus.

In der griechischen Literatur sind exotische Tiere Gegenstand der Betrachtung hauptsächlich von Reiseerzählungen, Historien oder Ethnographien, oder sie werden als Toponyme für Städte, Kolonien oder Landschaften eingesetzt. Zumeist behalfen sich die antiken Schriftsteller bei der Benennung fremder Arten mit einer geographischen

Ursprungsangabe:

\footnotetext{
${ }^{4}$ Hdt. 9,11
} 
Beispielsweise meint persikos ornis, der persische Vogel, den $\mathrm{Pfau}^{5}$ oder das Haushuhn. Auch die Morphologie oder das Verhalten des fremden Tieres wurden $\mathrm{zu}$ ihren Bezeichnungen: So fand man im Griechischen für das Flusspferd nach Aussehen und Lebensweise kurzerhand den Namen hippos potamios, Pferd vom Fluss, den Varro in diesem Laut ins Lateinische übernahm. ${ }^{7}$

Eine intentionale Verbreitung von in der neuen Umgebung fremdartigen Tieren kann hypothetisch aus zwei wichtigen Formen des Transportes resultieren: Verbreitung durch gezielten Import oder Migration von Haustierstämmen mit der wandernden Bevölkerung. Im Fall von im Mesolithikum besiedelten mediterranen Inseln etwa impliziert ein etwa zeitgleicher Faunenwandel, dass die Einwanderer eigene Tiere mitbrachten. ${ }^{8}$ Diese waren zwar unbekannt in der neuen Umgebung, den Ankömmlingen selbst aber vertraut. Kulturelle Rezeption ist demnach ein wichtiger Bestandteil jeder Definition des „Exotischen“. Da uns lediglich Ausschnitte der Vergangenheit überliefert sind, können Rekonstruktionen der Wahrnehmung nur sehr eingeschränkt vorgenommen werden, insbesondere für archäologische Kulturen ohne schriftliche Tradition.

Eine verbindliche Definition soll daher an dieser Stelle nicht angeboten werden, lediglich eine Arbeitshypothese als terminus technicus: Demnach werden Tiere als exotisch erachtet, die durch menschliche Handlung in ein Gebiet außerhalb ihrer natürlichen Verbreitungs- und Migrationszone eingeführt wurden und dort Seltenheitswert genossen. Dies betrifft intentional verbreitete Tiere ebenso wie die unvermeidlichen Zivilisationsfolger Insekten, Kleinsäuger etc. - deren Verbreitung unbeabsichtigt erfolgte, darunter die Hausmaus Mus musculus, deren Verbreitung nach Europa mit Ausnahme von Kreta augenscheinlich erst ab dem 1. Jt. v. Chr. signifikant in Gang

\footnotetext{
${ }^{5}$ Aristoph. Av. 707, uraufgeführt 414 v. Chr.

${ }^{6}$ Varro Ling. 5,78.

${ }^{7}$ Bodson 2005, 454; 456-457.

${ }^{8}$ Schüle 1993.
}

kam?. Auf die Betrachtung dieser Kommensalen, wie auch auf die der Wasserlebewesen soll an dieser Stelle weitgehend verzichtet werden. $\mathrm{Da}$ die Haustierverbreitung vergleichsweise besser untersucht ist ${ }^{10}$ wird der Fokus hier zudem auf den größeren Wildsäugetieren und großen Reptilien liegen, weitgehend unter Aussparung der Rohstoffe von Elefant und Strauß.

\section{HANDELSNETZWERKE UND VERTEILUNGSMUSTER}

Die Einführung exotischer Tiere in unterschiedliche Gebiete Europas ist kein Phänomen speziell des 1 . Jt. v. Chr. Wir fassen sie spätestens seit der Ausbreitung des Neolithikums, zu dessen Neuerungen auf wirtschaftlichem Niveau unter Anderem auch neue Haustiere gehörten. Über Fernhandelskontakte gelangten Produkte fremdländischer Tiere oder die Tiere selbst in die entsprechenden Gegenden, wo sie die oftmals sogar schon eine Wertschätzung bei den empfangenden Gesellschaften genossen. So sind aus der Kupferzeit bereits Elefantenelfenbein und Straußenei ${ }^{11}$ von der Iberischen Halbinsel bekannt, seit der Bronzezeit auch von anderen nordmediterranen Fundstätten. Flusspferdelfenbein ist im bronzezeitlichen Griechenland belegt ${ }^{12}$. Der Hausesel fand spätestens im Verlauf der Bronzezeit seinen Weg in die mediterranen Länder auf europäischer Seite. Auch niedergegangene eigene Tierbestände mögen durch Importe ergänzt oder ersetzt worden sein, wie beispielsweise R. Matthews die Einführung von Zebus in die Levante aus solchen Gründen heraus annimmt ${ }^{13}$. Möglich, dass ebenso die Griechen auf Importe zurückgriffen, als der heimische Löwe in klassischer Zeit rar wurde. Für den unteren

\footnotetext{
${ }^{9}$ S. Cucchi, Vigne, Auffray 2005, 439-440.

${ }^{10}$ Z.B. Benecke 1994; Herre, Röhrs 1990.

1 Fragmente einer Straußeneierschale stammen aus der Siedlung von Los Millares, Prov. Almeria (um 2400-2000 v. Chr.). Publ. bei Peters, von den Driesch 1990, 70, 89 Tab. 2.

12 So sind etwa aus dem ägäischen Raum rü Bronzezeit 13 Straußeneierfunde überliefert (dazu Sakellarakis 1990)

${ }^{13}$ Matthews 2002.
} 
Molaren eines Löwen aus dem Athenaheiligtum von Tegea, Arkadien (klassische Zeit, 490/480-323 v. Chr.) ${ }^{14}$ ist meiner Ansicht nach bereits ein Import in Betracht zu ziehen, sofern kein Erbstück vorliegt. Die Raubkatze gehörte jedenfalls zu den Waren, die die Phönizier verschifften (Skyl. $112=$ GGM 1,94). Mündliche, schriftliche und bildliche Tradition mögen die Einheimischen mit bestimmten fremden Tieren in Kontakt gebracht haben, bevor sie mit den realen Wesen konfrontiert wurden. Dass künstlerische Nachweise bis in weit ältere Zeit als die archäozoologischen Belege zurückreichen, ist ein häufiges Phänomen. Dieser Umstand ist vorrangig auf unterschiedliche Erhaltungsbedingungen und die Forschungstradition zurückzuführen, erschöpft sich darin aber nicht. Der Verbreitung des Bildes von einer fremden Kreatur, sei es auch ästhetisch und ikonologisch verändert, sind nicht zuletzt weniger Grenzen gesetzt als der des an ein bestimmtes Habitat gebundenen realen Tieres. So scheint zu gelten, was Aristoteles $^{15}$ über den schweren Stand des Hausesels im kälteren nördlichen Klima aussagt, denn der gegen Kälte und Nässe empfindliche Esel gelangte bis zum Ende des 1. Jt. v. Chr. nördlich offenbar nicht bis zum 50. Breitengrad.

Dem archäozoologischen Fundbild nach bedeutet der Transfer von exotischen Tieren im 1. Jt. v. Chr, nach Europa vor allem eine Verbreitung der Haustiere Esel Equus asinus, Katze Felis silvestris f. catus und Haushuhn Gallus gallus $f$. domestica. Zahme, bzw. echte Wildtiere und ihre Rohstoffe sind dagegen - mit Ausnahme der Elfenbeine verschiedener Tierarten und Straußeneier - bislang nur in Einzelfällen belegt. Bemerkenswerterweise sind uns von der Iberischen- und der Apenninenhalbinsel für das 1. Jt. v. Chr. lediglich Importe von typischen Haustieren bekannt. Reste von Wildtieren kennen wir hauptsächlich aus dem griechischen Raum. In manchen Fällen wiederum ist unklar, ob die betreffende

\footnotetext{
${ }^{14}$ Ostby et alii 1994, 111; Poplin 1994, 315 Abb. 1 rechts.

${ }^{15}$ Arist. gen. an. 748a.
}

Fremdspezies eine autochthone Tierart ist oder durch massive Importe in die Wildfauna eingegliedert wurde. Das betrifft insbesondere das Vorkommen des Damhirsches in Europa. Die Haltung eines lebenden Wildtieres in menschlicher Obhut ist allerdings bislang nur für einen Berberaffen Macaca sylvanus aus Nordirland relativ sicher anzunehmen (s.u.).

\section{EXVOTO AUS GRIECHISCHEN HEILIGTÜMERN}

\subsection{Das Heraion auf Samos}

Eine bemerkenswerte Sammlung von Überresten exotischer Tiere ist aus dem Heraion auf Samos bekannt. Die Fauna der Fundstätte arbeiteten J. Boessneck und A. von den Driesch auf. Die Mehrzahl der betreffenden exotischen Funde lag im Südtemenos und im Altarbereich vergesellschaftet. Aus dem Südtemenos, Bereich O 14, stammt ein 1977 freigelegter, kompletter Hornzapfen mit Ansatz der Schädelkalotte einer Nordafrikanischen Kuhantilope Alcelaphus buselaphus buselaphus, weitere Hornzapfen- und Schädelbruchstücke fanden sich 1983/84 im Bereich O 14, Quadrat VI, Abhub 7. ${ }^{16}$ Schädelfragmente eines Nilkrokodils Crocodylus niloticus stammen ebenfalls aus VI/6 und 7. ${ }^{17}$ Zwei Unterkiefereckzähne vom Flusspferd Hippopotamus amphibius wurden 1965 bei der Verbreiterung der Schnitte O $14 / \mathrm{O} 15$ in der so genannten "vorrhoikischen Schwemmschicht" entdeckt, ${ }^{18}$ ein dritter 1977 in O 14, Schicht b $[\mathrm{c} / 12]^{19}$ (s.o. Kuhantilope). Auch unter den Knochenabfällen im 1983 ergrabenen Südost-Bereich des Heraions befanden sich Flusspferd-Unterkiefereckzähne: Zwei schlecht erhaltene Canini stammen aus VI/1-2, sowie zwei Fragmente von zwei weiteren Canini aus Fläche VII, P 13c

\footnotetext{
$16 \mathrm{Zu}$ den Resten der Art Alcelaphus buselaphus Boessneck, von den Driesch 1981, 245-247; Boessneck, von den Driesch 1983, 8.

${ }^{17}$ Boessneck, von den Driesch 1988, hier bes. 8-9.

${ }_{18} \mathrm{Vgl}$. Kopcke 1968, 250 und 303.

${ }^{19}$ Boessneck, von den Driesch 1981, 245 geben als Fundbereich Planquadrat $\mathrm{O} 14$ b 32 an (vgl. Furtwängler 1980, Beil. 7), Zur Stratigraphie in diesem Bereich s. Furtwängler 1980, Beil. 4.
} 
32/33 Pithos. ${ }^{20}$ Ergänzt wurde diese Fundgruppe durch zwei Bruchstücke eines Unterkiefereckzahns, der 1984 in Fläche $\mathrm{XV} / 6 \mathrm{zu}$ Tage gefördert wurde. Bereits 1955 waren zudem im Altarbereich des Heiligtums mindestens sechs weitere Bruchstücke von Flusspferdeckzähnen geborgen worden, ${ }^{21}$ gemeinsam mit Eierschalenfragmenten vom Strauß Struthio camelus $^{22}$.

Chronostratigraphisch werden die Belege in die früharchaische Zeit (7. Jh. v. Chr.) eingeordnet. Die am Altar entdeckten Flusspferdhauer- und Straußeneifragmente sind offenbar als Votivgaben deponiert worden. Dagegen ist die Fundlage der übrigen Objekte in einer dunklen lehmigen Schicht abseits des Tempels im Südtemenos sicherlich sekundär. Zwischen dem Ende des 8. Jh. v. Chr. bis gegen $580 / 570$ v. Chr. erstreckte sich in den Bereichen $\mathrm{O} 13$ bis $\mathrm{O} 14 \mathrm{um}$ ein archaisches Wasserbecken herum ein offen liegendes, morastiges und bewachsenes Gelände, das zur Beseitigung nicht mehr verwendeter Sakralgegenstände benutzt wurde. ${ }^{23}$ In diese Auenlandschaft wurden die Horn- und Schädelreste der Kuhantilope, die Überreste eines Krokodilschädels, einiger Flusspferdhauer und eine Schale der Riesenmuschel Tridacna maxima wohl als ausgediente ex voto entsorgt. Zahlreiche Artefakte aus dem vorderorientalischen, westphönizischen, zyprischen und ägyptischen Bereich waren mit ihnen in den Südtemenos entfernt worden waren ${ }^{24}$.

\subsubsection{Kuhantilope}

\section{Einen komplett erhaltenen} Hornzapfen mit Kalottenrest aus dem Südtemenos (Abb. 1) wiesen die Bearbeiter J. Boessneck und A. von den Driesch morphometrisch einer voll ausgewachsenen Kuhantilope Alcelaphus buselaphus zu und vermuteten eine Zugehörigkeit zur

\footnotetext{
${ }^{20}$ Boessneck, von den Driesch 1988, 8 .

${ }^{21}$ Nachträglich bestimmt durch Boessneck und von den Driesch 1983.

${ }_{22}$ Boessneck, von den Driesch 1983, 21.

${ }^{23}$ Furtwängler 1980, 158.

24 Zusammengestellt sind die Fundpositionen der Artefakte aus Bereich O 13-O 14 bei Kopcke 1968, Beil. 8.
}

ausgestorbenen Unterart Alcelaphus buselaphus buselaphus ${ }^{25}$ (Abb. 2) - der damals in den Halbwüsten und Savannen Nordafrikas von Marokko bis nach Ägypten und der Levante bis Syrien verbreiteten Nordafrikanischen Kuhantilope. In den Schriftquellen wird die Kuhantilope von Herodot, Aristoteles, später von Plinius, Aelian und weiteren erwähnt und kam im Altertum in der libyschen Wüste häufig vor, wurde in der klassischen Periode als bubalus bezeichnet. ${ }^{26}$ Noch zur Zeit des Polybios (um 200-120 v. Chr.) waren riesige Herden solcher Tiere in Nordafrika verbreitet. ${ }^{27}$

Der komplett erhaltene samische Hornzapfen misst $33 \mathrm{~cm}$ entlang der Windung. Legt man die Feststellung von $\mathrm{C}$. Lenz zugrunde, dass die Hornzapfen von Kuhantilopen etwa $80 \%$ der tatsächlichen Gehörnlänge ausmachen, ${ }^{28}$ müsste das Horn des samischen Tieres rund $41 \mathrm{~cm}$ lang gewesen sein und kommt damit den $40 \mathrm{~cm}$ Länge nahe, die Lenz für zwei Exemplare der nordafrikanischen Unterart rekonstruiert. Da der Hornzapfen einem voll ausgewachsenen Individuum gehört, kann als sicher gelten, dass es sich tatsächlich um keine andere Unterart der Kuhantilope handelt: Die kleinste Unterart besaß auch das kleinste Gehörn, während die mit Blick auf die Horngestaltung morphologisch am nächsten stehenden rezenten Vertreter von Alcelaphus buselaphus aus der so genannten Westlichen Gruppe der Kuhantilopen Hörner von im Mittel $60 \mathrm{~cm}$, maximal 70 $\mathrm{cm}$ Länge ausbilden. ${ }^{29}$ Bruchstücke eines weiteren im Heraion von Samos entdeckten Hornzapfens sind zu kleinteilig, um Alter und Geschlecht des Individuums ablesen und die Hornlänge rekonstruieren zu können. ${ }^{30}$ Sie könnten ihrer Fundposition nach zu dem durch den vollständigen Hornzapfen repräsentierten Tier gehören.

\footnotetext{
${ }^{25}$ Zum Fund Boessneck, von den Driesch 1981, 245247.

${ }^{26}$ Keller 1909, 294.

${ }^{27}$ Keller 1909, 294.

${ }^{28}$ Lenz 1951, 30.

${ }^{29}$ Dorst, Dandelot 1973, 153-154.

${ }^{30}$ Zum Fund Boessneck, von den Driesch 1983, 8.
} 
Der Hornzapfen ist eng um die Hornbasis abgesetzt worden. ${ }^{31}$ Lediglich das Gehörn gelangte also in das Heraion, wo es wahrscheinlich als eine Kuriosität oder Trophäe von hohem ideellen Wert geweiht wurde, ${ }^{32}$ die nicht verbrannt, sondern geraume Zeit aufbewahrt wurde. Die Erlegung dieses Wildes stellte an die Jäger eine besondere Herausforderung, denn Kuhantilopen vermögen mit Geschwindigkeiten von $70-80 \mathrm{~km} / \mathrm{h}$ zu flüchten. Sie waren auf diese Weise weitaus schneller als jeder berittene Verfolger. Nur mit höchster Geschicklichkeit oder List in Gestalt einer Falle ließen sich die Tiere stellen. Die Ägypter fingen Kuhantilopen etwa mit Wurfschlingen und hielten sie halbdomestiziert für Opferzwecke, sie standen allerdings nicht so im Vordergrund der Wildhaltung wie etwa die Dorkasgazelle Gazella dorcas oder etwa die Nordafrikanische Oryx Oryx gazella dammah. $^{33}$

\subsubsection{Nilkrokodil}

In Europa sind nur aus dem Heraion von Samos Reste eines Nilkrokodils bekannt, in Gestalt zahlreicher Knochenfragmente eines Schädels (vgl. Abb. 3). ${ }^{34}$ Bemerkenswerterweise fehlen sämtliche Zähne. Knochenvergleichen und statistischen Berechnungen nach gehören die Überreste einem ausgewachsenen, kapitalen Nilkrokodils von mindestens $5 \mathrm{~m}$ Länge. $^{35}$

Ursprünglich war das wechselwarme Reptil Crocodylus niloticus in den Süßwasservorkommen und Küsten Afrikas verbreitet $^{36}$ von Madagaskar und dem Kap der Guten Hoffnung bis Mauretanien, Algerien, Tunesien, Tripolis, sowie Ägypten, woher vom Alten Reich (ca. 2686-2160 v. Chr.) an eine Vielzahl mumifizierter Krokodile überliefert $\operatorname{sind}^{37}$. Sporadisch kam es wohl an der levantinischen Küste vor, von wo aus es bis

\footnotetext{
${ }^{31}$ Boessneck, von den Driesch 1981, 246.

${ }^{32}$ Boessneck, von den Driesch 1981, 247-248.

${ }^{33}$ Boessneck 1953, 27.

${ }^{34}$ Dazu Boessneck, von den Driesch 1988, 8-9.

${ }^{35}$ Boessneck, von den Driesch 1988, 9.

36 In den Einzelheiten zur Verbreitung s. Trutnau 1994, 226-227.

${ }^{37}$ S. etwa Gambart, Podvin 2009 für das Alte bis Mittlere Reich.
}

in die ins Mittelmeer strömenden Flüsse des Landes gelangte. Andeutungen bei Plinius und Strabon ${ }^{38}$ zufolge lebte es in Syrien in einer Region, die heute allgemein an der Flussmündung des Nahr es-Zerkā verortet wird. $^{39}$ In Syrien, ${ }^{40}$ Palästina, Israel und Jordanien ist das Reptil heute ausgestorben, wie auch auf den Komoren, Seychellen, auf Sizilien und am Unterlauf des Nil bis mindestens nach Luxor hinauf. In Ägypten scheinen sich die Tierbestände wohl aufgrund der mit dem Bau des AssuanStaudammes einhergehenden ökologischen Veränderungen ein wenig zu erholen. ${ }^{41}$

Das Krokodil war in der bronzezeitlichen Kunsttradition Griechenlands ein recht seltenes, aber bekanntes Motiv. Ikonographisch ist das Tier auf Kreta bereits für die Stufen Mittelminoisch IB-II (um 2000/1950$1750 / 1720$ v. Chr.) belegt. In der Reptildarstellung auf einem aus der Siedlung von Tsoutsouros geborgenen Jadeitsiegel erkennt Phillips die Gestalt eines Krokodils. ${ }^{42}$ Vom griechischen Festland, ebenso wie von den Inseln, sind Krokodilmotive vor allem aus den Stufen Spätminoisch IIIA-B (um 1400-1200 v. Chr.) bekannt, so etwa ein Elfenbeinkamm aus Theben in Böotien aus Spätminoisch IIIA2-Kontext. ${ }^{43}$ Durch gute Kontakte Griechenlands zu Ägypten dürfte auch die eine oder andere Geschichte über das Nilreptil weitergegeben worden sein. Im 5 . Jh. v. Chr. ordnet Herodot (Hdt. 2,35) das

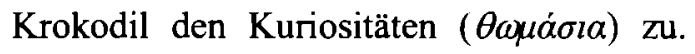
Womöglich besaß das Tier bei den Griechen bereits einen gewissen Ruf, als im 7. Jh. v. Chr. ein Krokodilschädel im Heraion auf Samos zu sehen war.

Für die Weihung eines lebenden Krokodils im Heraion von Samos gibt es

\footnotetext{
38 Strabon und Plinius nennen eine Stadt

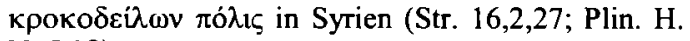
N. 5,18 ).

${ }^{39}$ So auch akzeptiert bei Phillips 2008,1, 207. Zu Kritik an der Verortung s. Lipiński 2004, 321 Anm. 289 mit weiterführender Literatur.

40 Syrien wird noch von Schmidt 1919, 419 als Verbreitungsgebiet des Nilkrokodils erwähnt.

${ }^{41}$ Trutnau 1994, 226.

42 Dazu Phillips 2008,1, 210 und Phillips 2008,2, 253 Kat.-Nr. 514.

${ }^{43}$ Phillips 2008,1, 211.
} 
keine Anhaltspunkte. Offenbar wurde der Krokodilschädel als eine solche kuriose Entdeckung, vielleicht gar als Jagdtrophäe aus Nordafrika mitgebracht. Die einst imposante Größe des Tieres unterstreicht den Trophäencharakter. ${ }^{44}$ Nicht auszuschließen ist, dass auch postkraniale Teile zu dem Votiv gehörten, die Haut etwa oder das ganze Tier. Doch sind weder die in der Haut liegenden Knochenplatten, noch postkraniale Knochen entdeckt worden. Auffällig ist das Fehlen der Zähne, die sich im Boden in der Regel besser als Knochen erhalten. Bei in Gefangenschaft gehaltenen Tieren scheint der Zahnwechsel oft gestört, Guggisberg berichtet von alternden Tieren, die große Zahnlücken aufwiesen. ${ }^{45}$ Von den 36 bis 38 Zähnen im Oberkiefer solcher Reptilien ist in diesem Fall allerdings kein einziger überliefert. Wahrscheinlich sind sie bei längerer Aufbewahrung des Schaustücks ausgefallen oder man brach bei der Entsorgung des unansehnlich gewordenen Stücks die Zähne aus - um sie beispielsweise in einer Steigerung des parspro-toto-Gedanken hinter der Präsentation als Kuriosität oder Trophäe noch länger aufzubewahren. Des Weiteren wäre zu überlegen, ob sie für einen anderen dekorativen oder mythologisch verklärten Zweck genutzt wurden, bzw. das Reptil durch das Entfernen der Zähne symbolisch unschädlich gemacht werden sollte.

\subsubsection{Flusspferdelfenbein}

$$
\text { Etliche Bruchstücke }
$$

von unbearbeiteten Flusspferdhauern aus dem Heraion auf Samos $^{46}$ sind besonders erwähnenswert. Waren während der Bronzezeit im griechischen Raum Flusspferdzähne neben Elefantenelfenbein ein geschätzter Rohstoff, ${ }^{47}$ sind

44 Dafür argumentieren auch Boessneck, von den Driesch 1988, 9.

${ }_{45}$ Guggisberg 1972, 60. Er schreibt diesen Umstand einer Fehlernährung und dem dadurch hervorgerufenem Kalziummangel zu.

${ }^{46} \mathrm{Zu}$ den Funden Boessneck, von den Driesch 1981, 245-248; Boessneck, von den Driesch 1983, 21-24; Boessneck, von den Driesch 1988, 8; Kopcke 1968, 303, Taf. 138,3-4.

47 Auf Kreta weist Caubet 2000, 121 zufolge die überwiegende Zahl von Flusspferdzähnen und daraus gefertigte Objekten im Verhältnis zu Elefantenelfenbein sogar auf eine bestimmte Präferenz ersterer Ressource hin. eisenzeitliche

Produkte

aus

Flusspferdzähnen selten. Nachdem gegen Ende der mykenischen Zeit bis in geometrische Zeit - etwa zum 9. Jh. v. Chr. - der ostmediterrane Raum offenbar ein Ausbleiben der Elfenbeinimporte und damit einen Niedergang der Elfenbeinverarbeitung erlebt hatte, ${ }^{48}$ entstanden erst im 1. Jt. v. Chr. syrophönizische Produkte in spätbronzezeitlicher Tradition und lebte der Transfer erneut auf. ${ }^{49}$ Offenbar aber verloren damals Flusspferdzähne ihre Bedeutung als Rohstoff. Die Fragmente von mindestens 14 unteren FlusspferdEckzähnen aus dem Heraion auf Samos sind die einzigen mir bekannten Objekte dieses Materials aus der vorchristlichen Eisenzeit. In diesem Zusammenhang ist vielleicht nicht unerheblich zu berücksichtigen, dass im Heraion auf Samos der orientalische Import - nämlich syro-phönizischer Elfenbeine - recht früh, bereits im letzten Viertel des 8. Jh. v. Chr. wieder einsetzte. Möglicherweise sind die Flusspferdhauer aus dem Heiligtum Relikte des Versuches, an die erstorbenen spätbronzezeitlichen Beziehungen bzw. Traditionen anzuknüpfen. Eine Erklärung, letztendlich afrikanisches Elefantenelfenbein $^{50}$ dem der Flusspferde vorzuziehen, könnte im starken Rückgang von Flusspferdpopulationen in deren natürlichen Verbreitungsgebieten in Palästina und vielleicht auch in Ägypten liegen, der den Export dieser Ressource limitierte. Spätestens seit dem 4. Jh. v. Chr. war die Art in Palästina ausgestorben. ${ }^{51}$ Allerdings stehen für die eisenzeitlichen Elfenbeinartefakte Europas größtenteils noch Analysen zur Identifizierung der

\footnotetext{
${ }^{48}$ Barnett 1948, 3; Krzyszkowska 1990, 112-113.

${ }^{49}$ Lafrenz 2003, 76.

50 In Ägypten selbst existierte der Afrikanische Elefant Loxodonta africanus bereits nicht mehr. Zu den letzten Nachweisen aus diesem Gebiet - Gräber 24 und 14 der Stufe Naquada IIA, B (um 3600 v. Chr.) der Nekropole von Hierakonpolis (HK6): Friedman 2003; Friedman 2004.

${ }_{51}$ Die späten Nachweise von Knochen und Zähnen stammen aus Tel Dor (11.-8. Jh. v. Chr.) unc früheisenzeitlichen Siedlungen Tell Garisa und Tell Qasile (Schichten des 12.-4. Jh. v. Chr.); Haas 1953, 32; Lafrenz 2003, 24-25.
} 
Tierart (z.B. durch Spektroskopie ${ }^{52}$ ) aus. Es ist daher zu früh, ernsthafte Spekulationen zur Frage des Exports anzustellen.

In Anlehnung an syrisch-phönizische Elfenbeinschnitzereien entstandene Produkte des 8 . Jh. v. Chr. sind aus der Idagrotte auf Kreta, aus Perachora und den drei mythischen Gründerorten auf Rhodos: Lindos, Kamiros und Ialysos dokumentiert. $^{53}$ Die frühzeitige Nachahmung zeigt ein lebhaftes Interesse der Griechen an der eigenen Herstellung solcher Gegenstände. Zweifelsohne blieb Elfenbein im Allgemeinen ein wertvoller Rohstoff für künstlerische Arbeiten ${ }^{54}$ und eine besondere Gabe für die Götter, ${ }^{55}$ sollten diese doch selbst die Schulter des Pelops durch eine elfenbeinerne ersetzt haben $^{56}$. Vielerlei Gegenstände, etwa Möbelbestandteile, Statuetten, Einlagen etc. wurden aus dem Rohmaterial gefertigt, jedoch im Gegensatz zum bronzezeitlichen Elfenbeininventar nur sehr wenige Siegel.

Ähnlich wie das Krokodil zählte Herodot auch das Flusspferd zu den Kuriositäten. ${ }^{57}$ Ein Flusspferdhauer ${ }^{58}$ aus dem Heraion auf Samos übertraf in Krümmungsgrad und Breite noch das größte vorhandene Vergleichsbeispiel eines starken Bullen ( 75 $\mathrm{cm}$ entlang der äußeren Krümmung, $2000 \mathrm{~g}$ Gewicht) $;^{59}$. Eine solche imposante Waffe kann, ähnlich dem Krokodilschädel oder dem Kuhantilopengehörn, auch einen Trophäencharakter besessen haben. Ein zweiter Hauer wird von J. Boessneck und A. von den Driesch in seiner äußeren Krümmung immerhin noch auf rund $50 \mathrm{~cm}$ Länge rekonstruiert. ${ }^{60}$ Ein dritter Hauer ist noch kleiner als die beiden früher

\footnotetext{
52 Zur Methodik u.a. Banerjee, Rodrigues 1993; Banerjee, Schneider 1996; Edwards et alii 1998; Shimoyama et alii 1998; Shimoyama, Morimoto, Ozaki 2004; Edwards et alii 2006.

${ }_{53}$ Borell 1978, 90.

${ }_{54}$ Barnett geht davon aus, Elfenbein habe zumindest bis ins 7. Jh. v. Chr. ein Luxusgut dargestellt (Barnett 1948, 24).

${ }_{55}$ Barnett 1948, 2.

56 Pind. Ol. 1,36-51.

${ }^{57}$ Hdt. 2,35 und 2,71.

${ }^{58}$ Kopcke 1968, 303; Boessneck, von den Driesch $1981,245$.

${ }^{59}$ Boessneck, von den Driesch 1981, 245.

${ }^{60}$ Boessneck, von den Driesch 1981, 245.
}

entdeckten Funde. ${ }^{61}$ Die Nutzung anderer Produkte als der Zähne, etwa der von Herodot für Ägypten berichteten Verarbeitung der Haut zu Speerschäften, ${ }^{62}$ ist für Europa jedoch nicht überliefert. Ein im Heraion entdecktes Knochenfragment auffälliger Größe (7. Jh. v. Chr.) ist nicht bis auf die Art herunter bestimmt, dürfte aber einem fossilen Hippopotamus gehört haben. $^{63}$ Antike Schriftquellen erwähnen bisweilen Knochen beeindruckender Maße, mit Ausnahme von Walknochen sind sie zumeist in einen mythologischen Kontext gestellt, als Knochen von Heroen oder Giganten. $^{64}$

\subsubsection{Straußeneier}

Im Fall von 20 Straußeneifragmenten vom Altar des Heraions auf Samos ist nicht festgestellt worden, ob es sich um Stückchen natürlich belassener Eierschale oder um die Bruchstücke eines Artefaktes handelte. D.S. Reese nimmt an, dass sie wie sämtliche übrige Belege aus dem griechischen Raum - zu rituell genutzten Gefäßen gehören. ${ }^{65}$ Die Befundlage gibt ihm insofern Recht, als dass für die vorchristliche Eisenzeit Reste von Straußeneiern bislang nur aus Heiligtümern dokumentiert sind, während Grabfunde nur sporadisch und in ihrer Zeitstellung unsicher überliefert sind, Funde aus Siedlungskontexten fehlen indes völlig. In den insgesamt neun griechischen Heiligtümern ist dabei eine Funktion der Straußeneigefäße rein als Votivgabe - ohne Weiternutzung im religiösen Kult - nicht ganz auszuschließen. Die Weihung von Straußeneiem und daraus gefertigten Artefakten ist im ostmediterranen Raum keine Seltenheit. ${ }^{66}$ Die Importe können aus Nordafrika und der Levante nach Griechenland gebracht worden sein.

\footnotetext{
${ }^{61}$ Boessneck, von den Driesch 1981, 246.

${ }^{62}$ Hdt. 2,71.

${ }^{63}$ Dazu Kyrieleis 1988, 220 mit Abb. 9.

${ }^{64}$ Kyrieleis 1988, 220-221. Zur Assoziation von Knochen mit Giganten und Monstem in der griechischen Antike etwa Thenius, Vávra 1996; Mayor 2000; Mayor 2001.

${ }^{65}$ Reese 2000, 402

${ }_{66} \mathrm{Zu}$ gleichzeitigen und früheren Beispielen von Zypern, aus der Levantine, Ägypten und Libyen: Reese 2000, 402.
} 


\subsection{5. Überlegungen zur Herkunft der} Funde

Der archäologische Befund spiegelt die Stellung von Samos als ein wichtiger Knotenpunkt im mediterranen Fembeziehungsnetz deutlich wieder. Über die sekundär zustande gekommene, heterogen zusammengesetzte Fundvergesellschaftung lässt sich die geographische Herkunft der Kuhantilopenhörner, der Straußeneierschale, ja selbst der Flusspferdzähne und des Krokodilschädels nicht näher zurückverfolgen. Ihre endemischen Verbreitungsgebiete dieser Zeit liegen sowohl in Nordafrika, als auch in der Levante. Eine ebenfalls in der einstigen Auenlandschaft des Südtemenos entdeckte Schale der Riesenmuschel Tridacna maxima, Form elongata, konnte nur im Schwarzen Meer gesammelt worden sein. Unter Berücksichtigung der im Importfundgut ein wenig überwiegenden Aegyptiaca - Gefäße aus ägyptischer Fayence und Statuen - lässt sich vermuten, dass die im Heraion repräsentierten Exotika eher aus dem ägyptischen Raum kamen. Nach J. Boessneck und A. von den Driesch spricht unter den politischen Gegebenheiten in der Saitenzeit (664-525 v. Chr.) das meiste für einen Austausch mit Ägypten. ${ }^{67}$ Zwar darf nicht vergessen werden, dass sich phönizische Präsenz unsichtbar hinter ihren eingetauschten Handelsobjekten verbergen kann, die dann einen originalen Ursprung suggerieren, ohne dass zwingend ein direkter Kontakt zwischen Herkunfts- und Fundregion bestand. Im Fundbereich weist aber nur ein westphönizischer $\mathrm{Kamm}^{68}$ auf phönizische Beziehungen hin, weshalb meines Erachtens gerechtfertigt ist, von Ergebnissen griechischer Kontakte zu Ägypten auszugehen.

\subsection{Das Artemis-/Apollonheiligtum bei Kalapodi}

Eine Rarität aus dem griechischen Raum ist das unscheinbare, verbrannte Hornzapfenfragment einer Dorkasgazelle Gazella dorcas (Abb. 4 und 5) aus dem Artemis- und Apollonheiligtum bei

\footnotetext{
${ }^{67}$ Boessneck, von den Driesch 1983, 22

${ }^{68}$ Kopcke 1968, Beil. 8 Fundpunkt 25.
}

Kalapodi, Phokis (Mittelgriechenland) ${ }^{69}$. Das Fundstratum aus braungelbem Lehm bildete eine Füllschicht über einem aschehaltigen Stratum voller verbrannter Knochensplitter und übrigem Brandschutt der beiden früharchaischen Tempel oder anderer Gebäude. Nach R.C.S. Felsch ist der Beleg in nachgeometrische Zeit, aber nicht in eine Zeit nach den Perserkriegen einzuordnen, ${ }^{70}$ dürfte also in archaischer Zeit (690-480 v. Chr.) anzusiedeln sein.

In den mit Akazien bestandenen Steppen und Halbwüsten Nordafrikas und der Levante streiften Dorkasgazellen frei umher, bei den Ägyptern zählten sie zudem zu den beliebtesten halbdomestizierten Tieren, die zu Opferzwecken in Gefangenschaft gehalten wurden. ${ }^{71}$ Die Gazellenhaltung ist außerdem mindestens für den Nordiran nachgewiesen. ${ }^{72}$ Die deutliche Krümmung des Homzapfens aus Kalapodi spricht eher für ein nordafrikanisches Exemplar mit lyraförmigem Gehörn als für ein levantinisches mit fast gerade geformten Hörnern $^{73}$, insofern das kalzinierte Fragment durch den Brand nicht unförmig verzogen ist. Demnach ist eine Verbindung zum ägyptischen, respektive dem von griechischen und phönizischen Kolonien dominierten nordafrikanischen Raum fassbar.

Die näheren Umstände, durch die der Hornzapfen verbrannte, lassen sich nicht zweifelsfrei klären, da der Fund in einer über ein Aschedepot aufgebrachten Füllschicht lag. Nicht auszuschließen ist, dass er sekundär aus einem anderen Bereich des Heiligtums in seine Fundlage eingetragen worden ist, etwa im Zuge der Aufschüttung der Füllschicht. Die Brandspuren lassen mehrere Deutungsmöglichkeiten zu. Einmal könnte das Gehörn/der Hornzapfen intentional den

\footnotetext{
${ }^{69}$ Das Fragment stammt aus Befund 13273 in Fläche L 26, wo es zwischen Quadratmeter 58 und 59 aus einem $10 \mathrm{~cm}$ breiten, in West-Ost-Richtung angelegten Profilsteg geborgen wurde. Publ. bei Stanzel 1991, hier bes. 104 und Taf. 1,3.

${ }^{70}$ Schriftliche Mitteilung von R.C.S. Felsch vom 01.03 .2010 .

${ }^{71}$ Boessneck 1988, 42.

${ }^{72}$ Brentjes 1965, 103.

${ }^{73}$ Uerpmann 1987, 94.
} 
Flammen übergeben worden sein. Ein Trophäenkult - zumindest als final im Heiligtum praktizierte kultische Handlung läge in diesem Fall nicht vor, da das Objekt bei dem Vorgang kleinteilig und unansehnlich verbrannt ist. Ein Weihopfer wäre wahrscheinlicher. So meint Stanzel, ein Besucher des Tempels habe den Hornzapfen von einer Reise mitgebracht oder erwarb ihn, um ihn als Weihegabe darzubringen. ${ }^{74}$ Vielleicht richtete er sich dabei an die Jagdgöttin Artemis. Der Hornzapfen, respektive das Gehörn mag dabei als pars pro toto des Opfers eingesetzt worden sein, denn weitere Skelettelemente der Dorkasgazelle sind nicht nachgewiesen.

Die Verbrennung des Belegs kann allerdings auch unbeabsichtigt, durch eine Feuerkatastrophe, erfolgt sein. Diese Möglichkeit ist in Betracht zu ziehen aufgrund des Brandschutts, über dem die Füllschicht angelegt wurde. Um 480 v. Chr. wurde bei Angriffen durch die Perser das Heiligtum zerstört, zwei im 7. Jh. v. Chr. angelegte Tempel brannten dabei nieder. ${ }^{75}$ Der Hornzapfen mag im Zuge von Aufräumarbeiten im Heiligtum in die Fundschicht gelangt sein. Fiel der Beleg einer solchen Feuersbrunst zum Opfer, ist vorauszusetzen, dass das Gehörn oder der Balg des Tieres in einem der Tempel aufbewahrt worden war. Hier ist eine Deutung als primär unverbrannte Weihegabe wahrscheinlicher. Für die Überführung eines lebendigen Tieres in das Heiligtum schließlich gibt es aufgrund fehlender Knochenfunde keine Anhaltspunkte. Im Fall eines olympischen Opfers - und nur zu diesem Zweck wäre der Import des lebendigen Tieres in das Heiligtum sinnvoll - wäre der Erkenntnis von Meuli zufolge der Schädel und manchmal nur das Gehörn des betreffenden Tieres des Tieres unverbrannt beim Altar, zumindest im Bezirk des Heiligtums belassen worden. ${ }^{76}$ Auch diese Deutungsmöglichkeit bedarf der

\footnotetext{
${ }^{74}$ Stanzel 1991, 104.

${ }^{75}$ Felsch 1987,13 und 24.

${ }^{76}$ Meuli 1946, 220.
}

unbeabsichtigten Verbrennung des Hornzapfens als zweiten Ereignisses.

In der griechischen Literatur werden

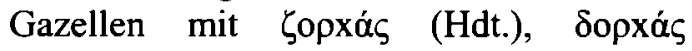

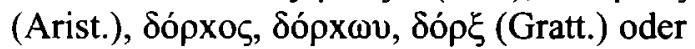
$\zeta o ́ \rho \xi$, bezeichnet, im Lateinischen als damma oder dorcas. ${ }^{77}$ Die scheuen (Dorkas-)Gazellen seien langsamer als Wildesel, berichtet Arrian, und man jage sie $\mathrm{zu}$ Pferd und mit Hunden unter Verwendung von Netzen, Lassos, Pfeilen und Speeren. ${ }^{78}$ In Ägypten und später im Römischen Reich wurden sie angebunden oder in Gehegen gehalten, ${ }^{79}$ als Delikatessen für die verwöhnten Mägen der Reichen. $^{80}$ Ihre gekrümmten Hörner konnten in Werkzeuge umgearbeitet werden und die Häute in teures Leder. ${ }^{81}$ Ihre Astragali wurden zur Würfelherstellung geschätzt. ${ }^{82}$ Alles in allem wird der Dorkasgazelle in der Literatur mehr Beachtung geschenkt, als der archäologische Befund widerspiegelt. Die griechische und römische Kunst kennt überreichlich viele Gazellen- und Antilopendarstellungen. Der Löwe und die Gazelle, wahlweise auch der phantastische Greif und die Gazelle finden sich beispielsweise häufig als archaisches Kunstmotiv. ${ }^{83}$ Doch ist es oft schwer möglich, die dargestellten Tiere bis auf die Art herab zu bestimmen. In der Kunst sind als Dorkasgazellen immerhin bereits im mykenischen Bereich zu finden: Die Darstellungen auf einer Dolchklinge und einer Malerei aus Thera ${ }^{84}$ dürften die kleine Gazellenart meinen.

\subsection{Messene}

Aus Griechenland stammt aus der Siedlung Messene, der Hauptstadt des antiken Messenien, der Nachweis einer Damagazelle Nanger dama ${ }^{85}$ (Abb. 6). Das zweite Zehenglied eines Tieres wurde im Fundbereich XVI/2 $/ 17$ NO9 am Altar des Demeter-Heiligtums in der Siedlung

\footnotetext{
${ }^{77}$ Hünemörder 2004, 216.

${ }^{78}$ Arr. Cyn. 24,1.

${ }^{79}$ Col. $9,1,1$.

${ }^{80}$ Juv. 11,121

${ }^{81}$ Hünemörder 2004, 716.

${ }^{82}$ Luc. Amores 16; Ath. 5,194a.

${ }^{83}$ Keller 1909, 289.

${ }^{84}$ Trantalidou 2000, Anm. 15.

${ }^{85}$ Zum Fund Nobis 2001, hier bes. 102-103.
} 
entdeckt. Chronostratigraphisch wird der Beleg in hellenistische Zeit (3.-2. Jh. v. Chr.) datiert. ${ }^{86}$

Innerhalb von Messene ist die Lage des Demeter-Heiligtums nicht sicher geklärt. ${ }^{87}$ Zwei Weiheinschriften befanden sich nicht in situ, inhaltlich könnten sie auf einen größeren Bereich um die nordwestliche Agora als Demeter-Heiligtum deuten. Dagegen lässt Pausanias Beschreibung der Örtlichkeit $^{88}$ auf eine Lage nahe des Dioskurenheiligtums schließen. Der Rundaltar selbst und ein Statuenfragment der Demeter wurden zwischen dem so genannten Hierothysion und dem Gymnasion entdeckt. Müth hält letzteren Ort jedoch als am wenigsten aussagekräftig für die Lokalisierung des Heiligtums, denn der Rundaltar und das Statuenfragment könnten sekundär verlagert sein wegen der allgemein in Messene zu beobachtenden Tendenz zur Materialverbringung von Norden nach Süden.

Der Inhalt einer Weihinschrift des späten 3. oder dem 2. Jh. v. Chr, aus Messene lässt auf einen zu dieser Zeit praktizierten Mysterienkult der Demeter schließen, da darin von einem Bankett im Rahmen von Mysterienzeremonien die Rede ist. ${ }^{89}$ Die Mysterien waren geheime Riten, die nur Eingeweihten zugänglich waren. Ein besonders seit dem 4. Jh. v. Chr. in eleusischem Kontext bekannter ikonographischer Typus, ${ }^{90}$ der Demeter auf einer mit einem Tierfell bedeckten cista mystica thronend zeigt, veranschaulicht den Mysterienaspekt. Die cista mystica war ein zylinderförmiger Behälter, in dem die im Kult der Demeter verwendeten Opferutensilien aufbewahrt wurden. Das Motiv erscheint sowohl auf Reliefs, Rundplastiken, attischen bemalten Pinakes, als auch Münzen und dergleichen. Den Kult der Demeter soll Kaukon der mythischen Stammesgründerin Messene gebracht haben, demzufolge ihre Verehrung dort genauso alt ist wie der erste Staat der Messenier. ${ }^{91}$ Die Siedlung lag an dem Platz,

\footnotetext{
${ }^{86} \mathrm{Vgl}$. Nobis 2001, 96 Tab 1

${ }^{87}$ Im Folgenden Müth 2007, 140-141.

${ }^{88}$ Paus. 4,31,9.

${ }^{89}$ Müth 2007, 140.

${ }^{90}$ Müth 2007, 66.

${ }^{91}$ Müth 2007, 131.
}

wo während eines Helotenaufstands der Messenier gegen die Herrschaft der Spartaner die Dissidenten fast ein Jahrzehnt lang bis zu ihrer Kapitulation um 445 v. Chr. belagert worden waren. Die unterlegenen Aufständischen erhielten freien Abzug und viele wanderten aus, etwa nach Tyndaros auf Sizilien oder Kyrene, der Hauptstadt der Cyrenaica in Nordafrika. Von der Überlieferung der Festordnung der Demeter sollte abhängen, ob die Messenier nach ihrer Vertreibung ihr Land wiedererlangen konnten. Dieses Dokument soll einst Aristomenes vergraben und der Thebaner Epiteles auf Ratschlag eines Hierophanten - oberster Priester der Demeter in Eleusis - wieder gefunden haben, der auch dem Thebaner Epaminondas (um 418-362 v. Chr.) befahl, den Messeniern ihr Land zurückzugeben. ${ }^{92}$ In der Schlacht von Leuktra $371 \mathrm{v}$. Chr. befreite Epameinondas das messenische Gebiet. Bei der Gründung der Stadt wurde daher auch Demeter als einer der ,großen Göttinnen“" geopfert. ${ }^{93}$

Der Demeterkult verknüpft auffällig stark politische und religiöse Aspekte. ${ }^{94}$ Es liegt nahe anzunehmen, dass der Fußknochen im Fell einer Damagazelle steckend als Weihung an Demeter in ihrer Funktion als Bindeglied zwischen dem alten und dem neuen, wiederbefreiten Messene gestiftet wurde. Nobis entwirft eine Vorstellung, wie sich die Nachricht von der Befreiung und der Gründung einer neuen Hauptstadt verbreitete und viele Emigranten die Gelegenheit zur Rückkehr ergriffen haben mögen: Vielleicht brachte ein Asylant aus Kyrene die in der Sahara und Sahelzone Nordafrikas lebende Damagazelle aus der neuen in die alte Heimat mit und opferte sie der Göttin Demeter zum Dank für die gewährte Rückkehr. ${ }^{95} \mathrm{Da}$ von der zweiten Stadtgründung von Messene 369 v. Chr. bis zur im 3./2. Jh. v. Chr. stattgefundenen Deponierung des Gazellenopfers ein längerer Zeitraum verstrich, können neben

\footnotetext{
${ }^{92}$ Paus. IV, 1,5. 20,4 27,7-8 und 33,5.

${ }^{93}$ Paus. IV,27,6.

94 So auch Deshours 2004, 121-122 und Müth 2007, 131.

${ }^{95}$ Nobis 2001, 103 und 105.
} 
der direkten Parallele zu einem im Zuge des Helotenaufstandes aus Messenien Vertriebenen und Zurückgekehrten auch anderweitige Assoziationen hergestellt werden. Es besteht beispiesweise die Möglichkeit, dass in späterer Zeit ein Kyrener oder Messener dem Ereignis mit einem Dankopfer gedachte. Der einzelne Fußknochen der Damagazelle mag zum Opferungszeitpunkt einem lebenden Geschöpf gehört, wahrscheinlicher aber im Balg des Tieres gesteckt haben, da nur ein distaler Gliedmaßenknochen überliefert ist. Vielleicht diente das exotische Fell sogar dazu, eine cista mystica zu bedecken.

In antiken europäischen Schriftquellen und Kunstwerken tritt die Damagazelle nicht auf. Die Ledragazelle Antilope damma, ${ }^{96}$ ein Synonym der Damagazelle bei $O$. Keller, ist ihm selbst von ägyptischen Darstellungen nur in geringer Zahl bekannt. Keller gewann den Eindruck, dieses Tier sei nur selten nach Ägypten gekommen und schwerlich nach Europa.

\section{DER DAMHIRSCH IN EUROPA -} ENDEMISCH ODER IMPORTIERT?

Das ursprüngliche Vorkommen des Damhirsches ist in der Forschung umstritten. Bislang lässt sich nicht mit Sicherheit entscheiden, ob diese Hirschart im mediterranen Raum - ausgenommen der östlichen Regionen von Griechenland hinauf bis nach Rumänien - im 1. Jt. v. Chr. ein exotisches Tier war oder nicht.

Maßgeblich sind zwei Arten dieses Hirsches zu unterscheiden: der kleinere Europäische Damhirsch Dama dama mit handförmigem Geweih und der tendenziell größere Mesopotamische Damhirsch Dama mesopotamica, dessen Geweih weniger handförmig ausgebildet ist. Bevorzugtes Habitat beider Arten sind bewaldete Gebiete. Obwohl der Damhirsch nie domestiziert wurde, ist er einfach zu zähmen. Das autochthone Herkunftsgebiet lässt sich heute schwer ausmachen, da die Hirschform seit römischer Zeit vom Menschen in extensivem Ausmaß verschleppt und verbreitet wurde. In der

${ }^{96}$ Keller 1909, 290.
Literatur herrscht wenig Konsenz über ihren Ursprung. J. Boessneck nannte den Mesopotamischen Damhirsch als Wildtier in Nordostafrika. ${ }^{97} \mathrm{~J}$. Clutton-Brock nimmt ein Wildvorkommen des Europäischen Damhirsches im gesamten mediterranen Bereich, möglicherweise einschließlich Nordafrika an, während der Mesopotamische Damhirsch in Westasien heimisch gewesen sei. ${ }^{98}$ Hingegen sehen W. Herre und M. Röhrs nur Kleinasien als endemisches nacheiszeitliches Verbreitungsgebiet des Damhirsches an, von wo aus dieser durch die Phönizier und Römer in den Mittelmeerraum eingeführt worden wäre. ${ }^{99}$ Die Hypothesen lassen sich schwer gegeneinander abwägen, da sie zum Teil auf Altfunden aufbauen, die entweder als gegeben akzeptiert oder im Gegensatz verworfen wurden ${ }^{100}$. Häufig wird auch in neuerer Zeit bei der Auseinandersetzung mit prähistorischen Damhirschknochen davon ausgegangen, der Damhirsch sei eine in seinem heutigen Verbreitungsgebiet vielleicht mit Ausnahme der Inseln einheimische Tierart, sodass wenig Augenmerk darauf gelegt wurde, den Fundzusammenhang auf Aspekte eines Imports hin zu untersuchen. Falsche Artenbestimmungen erschweren den zweifelsfreien Nachweis. ${ }^{101}$ Immerhin konnte die ernüchterte Feststellung von D. Ringe, es gäbe keinen einwandfreien nacheiszeitlichen Nachweis für die Existenz von Damhirschen in Europa, ${ }^{102}$ für Osteuropa revidiert werden. Inzwischen liegen zahlreiche Knochenfunde vor, die das Vorkommen von Dama dama in Griechenland frühestens seit dem $\begin{array}{lll}\text { Aurignacien } \quad(35.000-29.000 & \text { BP })\end{array}$ belegen. ${ }^{103}$ Auch in Bulgarien scheint der Europäische Damhirsch kontinuierlich im Pleistozän und Holozän verbreitet zu sein. ${ }^{104}$ In Rumänien stellen seine Reste aus

\footnotetext{
${ }^{97}$ Boessneck 1988, 39.

${ }^{98}$ Clutton-Brock 1999, 203.

${ }^{99}$ Herre, Röhrs 1990, 72.

100 So etwa Ringe 1959, dessen Ansichten sich allgemein durchgesetzt haben.

$101 \mathrm{Vgl}$. Sykes 2004, 75.

${ }^{102}$ Ringe $1959,23-28$.

${ }^{103}$ Zur Verbreitung des Damhirsches in Griechenland zuletzt Yannouli, Trantalidou 1999

${ }^{104}$ S. dazu Ninov 1999, 333-337.
} 
neolithischen und chalkolithischen Fundstätten noch keine allzu große Seltenheit dar, die Region dürfte aber wohl die nördliche Verbreitungsgrenze gebildet haben. ${ }^{105}$ Für Mitteleuropa nennt W. von Koenigswald Funde der römischen Kaiserzeit aus Trier und Augst als einige der wenigen eisenzeitlichen Belege nördlich der Alpen. ${ }^{106}$ Er merkt an, erst im Mittelalter sei der Damhirsch in Mittel- und Nordeuropa in solchen Zahlen importiert worden, dass dies Einfluss auf den Wildbestand hatte. Von der Iberischen Halbinsel ist der Damhirsch nacheiszeitlich erst aus der frühen nachchristlichen Zeit bekannt und scheint damit durch die Römer eingeführt worden zu sein, ${ }^{107}$ ebenso wie im nördlichen Frankreich ${ }^{108}$.

Im Bereich der Inseln findet sich der Damhirsch offenbar seit neolithischer Zeit auf Zypern, wo aus entsprechenden Schichten von Khirokita und Erimi von Funden berichtet wird. ${ }^{109}$ Aus der Siedlung von S'Illot, San Lorenzo, Mallorca stammen Damhirschknochen aus oberflächlichen Schichten mit römerzeitlichen Einlagerungen. ${ }^{110}$ Aus Großbritannien gehören erste Nachweise ebenfalls römischer Zeit an. ${ }^{111}$ So betrachtet handelt es sich bei Knochenfunden in der Nähe Siziliens eher um Importe: Aus der phönizischen Siedlung auf Motya (etwa 8.4. Jh. v. Chr. $)^{112}$, einer nur $800 \mathrm{~m}$ langen und $600 \mathrm{~m}$ breiten Insel vor Siziliens Westküste, sind mehrere Reste vom Damhirsch überliefert. ${ }^{113}$ Auch ein während

105 Bălășescu, Radu 2004, 173-174; Haimovici 2007, 298.

${ }_{106}$ Von Koenigswald 2002, 85.

$107 \mathrm{Zu}$ den betreffenden zwei Nachweisen der römischen Kaiserzeit aus São Pedro Fronteira und Torre de Palma in Portugal (1.-5. Jh. v. Chr.) und anderen frühen Belegen aus dem mediterranen Raum Davis, MacKinnon 2009. Ein Damhirschknochen von Torre de la Palma, Schicht 2 ist radiokarbondatiert in die Zeit zwischen dem 1.-3. Jh. n. Chr. (Davis, MacKinnon 2009, 16).

${ }_{108}$ Pascal, Vigne, Lorvelec 2003.

${ }^{109}$ Clutton-Brock 1999, 203 nach King 1953.

${ }^{110}$ Uerpmann 1971, 9-10.

111 Sykes 2004, 79.

112 Zur Fundstätte Isserlin, Du Plat Taylor 1974.

113 Erwähnt bei Ryder (Ryder 1975, 213-215) mit Verweis auf einen Beitrag bei Isserlin, Du Plat Taylor (1974), der allerdings nicht erschienen ist. Davis, Mackinnon 2009 nehmen keinen Bezug auf die Funde und geben als älteste nachneolithische Belege der Jahre 1972-1973 nördlich von der Küste von Isola Lunga untersuchtes punisches Schiffswrack, radiokarbondatiert in das 3. Jh. v. Chr., barg einen vollständig erhaltenen Mittelfußknochen von Dama dama. ${ }^{114}$ Der Lebensraum dieser Tiere auf der Insel muss so stark begrenzt gewesen sein, dass sie nur als zahme oder gefangene Tiere dort hatten leben können oder sogar nur ihre Rohstoffe auf die Insel gebracht wurden. Wir wissen nicht, ob das punische Schiff einen Bearbeitungsspuren aufweisenden Knochen als Werkstoff auf eine solche Insel einführen, oder von dort fortbringen sollte. In Anbetracht der in der Siedlung von Campanaio auf Sizilien (um 200 v. Chr. 460 n. Chr.) in großer Zahl geborgenen Knochen vom Damhirsch - im Fundmaterial stellten sie die zweithäufigste Tierart nach den Ovicapriden - zieht F. de Angelis in Erwägung, dieser Hirsch könne in Restbeständen auf Sizilien und in Süditalien nacheiszeitlich noch heimisch gewesen sein. ${ }^{15}$ Allerdings ist der Damhirsch in genau diesen Regionen nur bis in neolithische Zeit nachgewiesen. ${ }^{116}$ Aus der Folgezeit sind Knochenfunde von Sizilien, offenbar Schlachtabfälle, erst des 3. Jh. v. Chr. aus Morgantina ${ }^{117}$ bekannt.

Problematisch ist auch die Beurteilung von Altfunden mehrerer Geweihfragmente aus bronzezeitlichen Feuchtbodensiedlungen in Italien, dem Bodenseebereich und aus Olmütz in Tschechien, die als Damhirschreste identifiziert wurden. D. Ringe fand keinen Zugang zu den verschollenen Objekten und war so gezwungen, die autochthone Verbreitung des Damhirsches in Frage zu

zwei Damhirschknochen von der Fundstelle Gravina in Italien (Mitte 2.-spätes I. Jh. v. Chr.) an (Davis, MacKinnon 2009, 23). Letzterer Befund hilft, das zeitliche Fundbild der Spezies zu verdichten. Ein Fortbestehen von Damhirschpopulationen im italischen Raum, eventuell stabilisiert durch Importe, liegt im Bereich des Möglichen, sodass die Römer vielleicht auf eigene Ressourcen zurückgreifen konnten.

${ }_{114}$ Ryder 1975, 213; 214 Abb. 1.

115 De Angelis 2001, 182.

${ }^{116}$ Davis, MacKinnon 2009, 23. Es handelt sich dabei um jeweils ein Metatarsus- und ein Geweihfragment von der Fundstätte Megara Hyblaea auf Sizilien (Villari 1986) und Masseria Valente, Apulien in Süditalien (Bökönyi 1983).

${ }^{117}$ Bell 1988, 329. 
stellen $^{118}$. Einer der frühen Finder, L.H. Jeitteles, verglich seinen Fund aus der bronzezeitlichen Feuchtbodensiedlung von Olmütz mit anderen Funden aus den italienischen Terramare $\quad-\quad$ zwei Geweihfragmenten aus der Feuchtbodensiedlung Modenese in der Emilia Romagna - und befand, dass sie zum Damhirsch gehörten - damals als Cervus dama bezeichnet. ${ }^{119}$ Leider standen die italienischen Funde, die damals in das Museum Modena gelangten, für Nachuntersuchungen nicht zur Verfügung. Nach wie vor ist unsicher, ob die Phönizier Damhirsche oder deren Geweihe und Häute (mitsamt den darin steckenden Mittelfußknochen) vom italienischen Festland aus verschifften, oder ob wir umgekehrt einen Import vor römischer Zeit fassen. Auffällig ist nur der für die vorrömische Zeit fehlende Beleg des Damhirsches auf der Iberischen Halbinsel, wohin durch die engen Kontakte zu den Phöniziern exotische Materialien und Tiere sonst stets frühzeitig und in beachtlichem Umfang gelangten. Sollten die Punier Damhirsche aus den reichen ostmediterranen Beständen in ihre Handelsstationen auf Sizilien eingeschifft haben, stünde dann nicht auch der Transport bis in den westmediterranen Raum zu erwarten? Wenn wir hier nicht mit einem Forschungsartefakt umgehen, gehörte womöglich die europäische Mittelmeerküste nicht zur Seite der Empfänger, sondern diente Motya wie ihr Pendant Kerne (Skyl. 112) auf nordafrikanischer Seite als Umschlagplatz von zum Export bestimmten Tauschwaren.

\section{DER BERBERAFFE IN IRLAND}

Bis in die mitteleisenzeitliche Siedlung von Navan Fort, County Armagh in Nordirland gelangte ein Berberaffe Macaca sylvanus ${ }^{120}$ (Abb. 7). Die befestigte, mehrphasige Siedlung (Bereich B) wies Grundrisse von ringförmigen

\footnotetext{
${ }^{118}$ Ringe 1959.

119 Jeitteles 1872, 30-31.

120 Zur Fundstätte Waterman 1997; dort speziell zum Fund des Berberaffen Napier, Jenkins 1997.
}

Hausstrukturen auf: Rundhäuser, bei denen als Besonderheit offenbar je eine „,nördliche“ und „,südliche“" Ringanlage eine zeitgleiche "Wohn"-einheit bildeten. Den Schädel des weitgereisten Artvertreters (Abb. 8) fand man in der Grabenanlage C2: dem zugeschütteten und sekundär gestörten Ringgrabenfundament einer aufgegebenen Hausstruktur. Aufgrund seines guten Erhaltungszustandes dürfte er nach Ansicht der Ausgräber Bestandteil der sekundären Verfüllung sein und gehört nicht zum gestörten älteren Material. Unweit davon lag der zugehörige Unterkiefer in einer Kulturschicht zwischen den Gräben N und $P$ (Phase 3 (ii-iii)). Über den Befundkontext in Kombination mit einer Nitrogenanalyse und einem $\mathrm{C}^{14}$-Datum des Schädels $(220-80 \text { v. Chr. })^{121}$ ließ sich der Zeitraum der Deponierung auf etwa 220-95 v. Chr. einschränken, mit einer höchsten Wahrscheinlichkeit um 150 v. Chr. ${ }^{122}$ P.H. Napier und P.D. Jenkins identifizierten ein etwa fünf- bis sechsjähriges, jung adultes männliches Individuum, dessen Schädel einen dem Wildtier vergleichbar kräftigen Schädelkamm mit niedrigen, rechteckigen Augenhöhlen und komprimierten Nasalknochen als Merkmal für in Gefangenschaft lebende Tiere kombinierte. ${ }^{123}$ Offenbar ist der Berberaffe als noch in der körperlichen Entwicklung befindliches Jungtier aus seinem natürlichen nordafrikanischen Artverbreitungsgebiet in Marokko, Tunesien, Libyen und Tunesien in die menschliche Obhut geraten und nach Irland verschifft worden.

Das dort herrschende eher raue nordirische Klima konnte der an gebirgige Landschaften angepasste Berberaffe im wettergeschützten Siedlungsbereich sicherlich aushalten. Indirekt nachgewiesen durch die starke Präsenz durch Eichelmast unterhaltener Schweine im archäologischen Befund ist ein Eichenbestand nahe der Fundstelle, der potentiell auch dem Affen Nahrung bot. Die Frage nach der Todesursache des Tieres rückt einen stark

\footnotetext{
${ }^{121}$ Warner 1997b, 192.

122 Lynn 1997a.

${ }^{123}$ Napier, Jenkins 1997, 120-121.
} 
abgenutzten oberen linken Eckzahn ins Blickfeld. Durch permanentes Schleifen über einen Gegenstand, vielleicht seinen Antagonisten, den nicht erhaltenen unteren ersten Backenzahn, ist der Markkanal eröffnet worden. Eindringende Bakterien könnten eine Zahnfachentzündung und in deren Gefolge eine Sepsis mit letalem Potential hervorgerufen haben. Insofern am Knochen entzündliche Reaktionen sichtbar sind, sollte ein solcher pathologischer Vorgang in Betracht gezogen werden. Allerdings äußern sich die Bearbeiter P.H. Napier und P.D. Jenkins nicht zum Zustand des Knochengewebes rund um den betroffenen Eckzahn.

Der Berberaffe von Navan Fort war augenscheinlich ein gezähmtes Wildtier. Welchen Kontakten aber verdankten die ansässigen Menschen das aus Nordafrika stammende Tier? Aus Navan Fort liegen Importe vor, die den Fundplatz mit Großbritannien und dem europäischen Festland verknüpfen: u.a. zinnhaltiges Glas, das man während des 2 . Jh. v. Chr. aus dem Nordwesten Europas bezog, woher auch eine latènzeitliche dekorierte Ringnadel stammen dürfte, ein Kamm aus Hirschgeweih besitzt vor allem großbritannische, aber auch kontinentale Parallelen. ${ }^{124}$ Keinerlei explizit mediterrane Importe sind überliefert. Wenden wir uns zunächst nach Großbritannien und nach dem Festland, um Parallelen zum Fund von Navan Fort zu prüfen, so ist der zeitnächste Fund ein Affenskelett von Dunstable, Bedfordshire in England aus dem 2. Jh. $\mathbf{n}$. Chr. ${ }^{125}$ Vom Kontinent soll schon aus vorrömischer Eisenzeit ein Berberaffe aus einer Deponierung im Bereich des treverischen Oppidum auf dem Titelberg stammen. ${ }^{126}$ Der Befund konnte jedoch nicht verifiziert werden. Es besteht zumindest die Möglichkeit eines Importes, den west- bis mitteleuropäische Reisende vermittelten. Nach P.H. Napier und P.D. Jenkins sprechen irische Kontaktfunde aus dem südiberischen Raum, wie Fleischhaken

\footnotetext{
${ }^{124}$ Wamer 1997a, 101

${ }^{125}$ Napier, Jenkins 1997, 121.

126 Napier, Jenkins 1997, 123 nach einer mündlichen Mitteilung von Ralph M. Rowlett.
}

und Kessel irischen Typs, für einen Austausch auch mit diesem Gebiet, wie auch die mobilen karthagischen Seefahrer, die an der nordafrikanischen Küste direkten Zugriff auf die Tierbestände hatten, als Exporteure in Frage kommen. ${ }^{127}$ Lynn resümiert, dass wohl im 2 . Jh. v. Chr. ein wie auch immer gearteter direkter oder indirekter Kontakt mit im mediterranen Raum Reisenden bestand. ${ }^{128}$

Die Rezeption des Berberaffen bei den Bewohnern der Siedlung von Navan Fort erschließt sich aus dem Fundkontext recht gut. Nach Lynn lassen die Baustrukturen und wenigen Funde in Fundstätte B von Navan Fort erkennen, dass sich in der Eisenzeit bereits ein regionales Machtzentrum an diesem Ort herausgebildet hatte, dessen Einwohner offenbar einen hohen Status in der Gesellschaft genossen. ${ }^{29}$ Er argumentiert für diese Deutung mit der Größe der Häuser und der Siedlungskontinuität in einer Abfolge von 12 Ringanlagen A1-E3. Eine überproportionale Menge an Schweineknochen als Küchenabfälle, sowie nicht zuletzt Importe als Repräsentanten von Fernhandelsbeziehungen, zu denen der Berberaffe selbst auch zählt, bieten Argumente für weitreichende Kontakte und Einfluss. Eine Deutung des Gesamtbefundes als sowohl rituell, als auch weltlich bedeutsamer $\mathrm{Ort}^{130}$ erklärt sich über die literarische Tradition: Navan Fort wird allgemein mit dem als Emain Macha bezeichneten Sitz der Ulster-Könige assoziiert, dessen Errichtung je nach Quelle unterschiedlich um $668 \mathrm{v}$. Chr. (Annals of the Four Masters (Anno Mundi 4532)), rund 450 v. Chr. (Annals of Clonmacnoise) oder um 307 v. Chr. (Annals of Tigernach and Inisfallen) angegeben wird ${ }^{131}$. Lässt man eine Identifikation des Ortes mit dem mythischen Emain Macha unberücksichtigt, so ist eine zumindest regional herausragende Stellung des Ortes archäologisch nachgewiesen. Der Berberaffe kann wohl kaum losgelöst von

\footnotetext{
${ }^{127}$ Napier, Jenkins 1997, 123.

${ }^{128}$ Lynn 1997b, 213.

${ }^{129}$ Lynn 1997b, 213.

${ }^{130}$ Wamer 1997a, 104.

${ }^{131}$ Vgl. Mallory 1997, 199.
} 
dem repräsentativen Gepräge Navan Forts betrachtet werden. Eine sakrale Konnotation lässt sich allerdings nicht genug begründet herausfiltern.

Dass der Affe als eine Besonderheit aufgefasst wurde, findet in der Tatsache Ausdruck, dass Schädel und Unterkiefer des Tieres im archäologischen Befund nicht im anatomischen Verband lagen. Diese Skelettelemente waren folglich über den Tod des Tieres hinaus eine Zeitlang aufbewahrt und vielleicht sogar in einem der Rundhäuser von Navan Fort ausgestellt worden. Zum Ende der Aufbewahrungsphase gelangten die Knochen in die Fundamentverfüllung der Ringanlage $\mathrm{C} 2$, respektive nahebei in eine zeitgleiche Kulturschicht. Die Deponierungsweise wirkt beiläufig und kann nicht als nachträgliche Bestattung oder auch nur pflegliche Niederlegung gelten, vielmehr als Entsorgung mitten in der Siedlungsstruktur. Der Schädel war - da ihm auch in situ mehrere vordere Zähne fehlten, die nicht aufgefunden werden konnten - vielleicht im Laufe der Zeit auch recht unansehnlich geworden.

Werfen wir einen vergleichenden Blick in die schriftliche und künstlerische Tradition der mediterranen Antike, so erscheint der Berberaffe in der griechischen und lateinischen Literatur vorwiegend als ein Gegenstand von Lächerlichkeit. In der bildlichen Kunst stellt er sich oft als gezähmtes, gar abgerichtetes Haustier dar, auch als Parodie eines Reiters zu Pferd, sehr häufig aber ist der Affe allein in gekauerter Haltung Gegenstand des künstlerischen Interesses. Eine religiöse Konnotation ist auch hier nicht ersichtlich.

Der späteisenzeitliche osteologische Beleg aus Navan Fort steht - nicht zwingend im Einklang mit dem negativen literarischen Leumund - in deutlich repräsentativem Zusammenhang. Der Berberaffe scheint sowohl dem Befundkontext, als auch dem paneuropäischen Kontext nach als possierliches zahmes Tier gehalten worden zu sein, Implikationen für eine religiöse Konnotation finden sich nicht. Der Beleg und womöglich ein Fund vom Titelberg, sowie kaiserzeitliche und mittelalterliche
Knochenfunde $^{132}$ zeigen, dass der Berberaffe als gelehriges Haustier und amüsante Attraktion über die Zeiten hinweg stetig wertgeschätzt wurde.

\section{HINWEISE ZUR REZEPTION DER IMPORTE}

Die Wahrnehmung der erworbenen Raritäten kann von der empfangenden Bevölkerung vollständig mit übernommen worden sein, wie sich etwa im Fall des Berberaffen von Navan Fort andeutet. Ebenso mögen selektiv nur bestimmte Elemente der Rezeption in Erfahrung gebracht oder beibehalten worden sein, wie es für die griechischen Weihegaben diskutiert werden kann: Fell, Hörner und Schädel fremder Tiere brachten die Griechen als besondere Votivgaben in ihre Heiligtümer, wo sie aufbewahrt und an bestimmten Orten ausgestellt oder an speziellen Festtagen gezeigt wurden.

Imitationen können auf eine besondere Wertschätzung der Vorbilder hinweisen, selbst wenn diese archäozoologisch gar nicht bekannt sind. Hirsche, Antilopen und Gazellen als zahme Reit- und Gespanntiere etwa sind ein geläufiges Motiv der griechischen und römischen Ikonographie. Auch archäozoologisch sind uns aufgezäumte Hirsche bekannt bemerkenswerterweise aus den gallorömischen Provinzen. Beispielsweise aus einer latènezeitlichen Nekropole von MontGravet bei Villeneuve-Renneville, Dép. Marne stammt das Skelett eines Rothirsches mit Spuren einer Aufzäumung am Unterkiefer. ${ }^{133}$ Am Geweih sind bis auf die Hauptstange alle Äste abgetrennt, was der Waffe das Aussehen eines Antilopenhorns verleiht. $^{134}$ Möglicherweise steht hinter diesem Erscheinungsbild, das wir beispielsweise auch vom Hirsch 143 aus der gallo-römischen Nekropole von Nogent-sur-Seine, Dép. Aube kennen, ${ }^{135}$ eine intendierte Imitation des griechisch-

\footnotetext{
${ }^{132} \mathrm{Vgl}$. die Nachweise bei Napier, Jenkins 1997, 121 für Großbritannien.

${ }_{133}$ Dazu Poplin 1993, 532-538.

134 Poplin 1993, 535-536 Abb. 4-5; Poplin 1996, 401-402.

${ }_{135}$ Zu den Hirschgräbern Poplin 1996; hier bes. 398 Abb. 4.
} 
römischen Kulturelementes, das in seiner Ursprungsregion selbst keine osteologischen Parallelen vorweisen kann.

\section{GABE UND GEGENGABE}

Ergänzende Hinweise auf den Transfer lassen sich in der jeweiligen Exportregion selbst finden. So ist beispielsweise auf Mogador vor Essaouria, Marokko, nebst unbearbeitetem und bearbeitetem Elefantenelfenbein, sowie Straußeneierschalenresten auch der Unterkieferknochen eines Atlaslöwenjungen entdeckt worden. ${ }^{136}$ Derzeit ist der auf der 500 bis $600 \mathrm{~m}$ lang gestreckten Insel verortete phönizische Handelsposten des 7. Jh. v. Chr. Teil eines Ausgrabungsprojekts des Deutschen Archäologischen Instituts. Punische Amphoren belegen den Fortbestand des Handelspostens bis ins 4. Jh. v. Chr. ${ }^{137} \mathrm{D}$. Marzoli und A. El Khayari nehmen an, dass die Knochen, Zähne, Hörner und Felle exotischer Tiere, gar lebende Tiere von dieser Insel aus in den mediterranen Raum verhandelt wurden - wie mutmaßlich das Löwenjunge auch für den Export gefangen worden war - und verweisen auch auf eine Passage in den Küstenbeschreibungen des Pseudo Skylax (Skyl. 112). ${ }^{138}$ Diese enthält die von einer älteren Quelle aus hellenistischer Zeit entlehnte Beschreibung der entfernten Insel Kerne, wo sich phönizische Händler niederließen, um mit den auf dem Festland lebenden Äthiopiem Handel zu treiben. Der Handel gestaltete sich folgendermaßen, dass die Äthiopier Felle von Haustieren wie wilden Tieren anboten, darunter Bälger von Hirschen, Löwen, Leoparden, ja sogar von Elefanten. Im Gegenzug boten die Phönizier Salböle, Edelsteine aus Ägypten, attische Keramik und Weinkrüge zum Tausch. Die überwiegende Zahl der phönizischen Keramik von Essouria weist in ihrer Magerung und Brenntechnik auf eine Herkunft aus Südspanien hin, ein Teil auf andere phönizische Stationen entlang der

\footnotetext{
${ }^{136}$ Marzoli, El Khayari 2009, 82 und 86.

${ }^{137}$ Marzoli, El Khayari 2009, 92.

${ }^{138}$ Marzoli, El Khayari 2009, 86.
}

Küste Afrikas. ${ }^{139}$ Eine in den griechischen und levantinischen Raum reichende Verbindung ist von der Fundstelle archäologisch noch nicht nachgewiesen. Allein schon Gadir an der südspanischen Küste war rund $900 \mathrm{~km}$ Schiffslinie entfernt. Ein leichter zu realisierender Transfer der Handelsware entlang der wie auf einer Perlenschnur aufgereihten phönizischen Faktoreien entlang der Mittelmeerküste ist auch eine Erklärungsmöglichkeit für das Fehlen von Produkten selbst aus dem phönizischen Mutterland.

Nicht in jedem Fall aber waren Importtiere primär Handelsware, sondern befanden sich etwa als Proviant oder Werkstoff mit praktischem Nutzen an Bord der Reiseschiffe. Damhirsche dienten den Phöniziern als Rohstoff- und Nahrungsressource, die sie vielleicht nicht auf die Insel Motya vor Sizilien brachten, sondern im Gegenteil von dort mitnahmen. Eher als Trophäen anzusprechen sind Belege der Dorkas-, der Damagazelle, sowie der Kuhantilope, und vielleicht auch der Schädel eines Nilkrokodils, die in griechischen Heiligtümern geweiht worden waren, um die betreffenden Gottheiten zu ehren und sich gewogen zu stimmen. Es ist auffällig, dass im griechischen Raum exotische Wildtiere eng mit dem religiösen Kult assoziiert auftreten. Grab- oder reine Siedlungsfunde fehlen praktisch vollständig. Das mag unter anderem mit den besseren Überlieferungsbedingungen in Heiligtümern zusammenhängen, wo einmal veräußerte Gaben in der Regel dauerhaft verblieben, im Gegensatz zum im Siedlungsbereich einzuordnenden privaten Eigentum. Der fehlende Nachweis dieser Importe auch im Grabkult hebt ihre sakrale Konnotation allerdings besonders hervor.

\section{BEDEUTUNG DER IMPORTE}

Häufig bleibt bei den archäozoologischen Nachweisen im Dunkeln, welche Intentionen hinter den Importen standen, ob es sich etwa um Mitbringsel, Proviant, Tauschgabe odur

${ }^{139}$ Marzoli, El Khayari 2009, 92. 
Anderes handelt. Der Import fremder Tiere oder Zuchtformen war nur dann von wirtschaftlicher Relevanz, wenn er in großem Maßstab stattfand und durch ihn die entsprechende Tierform langfristig in die bestehende Haustierwirtschaft ${ }^{140}$ oder Wildfauna integriert werden konnte. Sind Tiere in solchen Mengen eingetragen worden, dass sich stabile Populationen bildeten, so ist eher an Handel zu denken, wenn nicht von einer Initiative der ansässigen oder sich neu ansiedelnden Bevölkerung ausgegangen wird und unbeabsichtigt eingeschleppte Zivilisationsfolger, wie die Hausmaus, unberücksichtigt bleiben. Für das 1. Jh. v. Chr. trifft dies dem archäozoologischen Befund nach nur auf Haustiere zu. Ähnlich lassen sich die Rohstoffe Straußenei und Elefantenelfenbein werten, die im mediterranen Raum in solchen Quantitäten vorliegen, dass ihrer Verbreitung ein Handel zugrunde liegt und/oder eine standardisierte Form des diplomatischen Geschenkes. Dagegen bleiben die übrigen aufgeführten Belege von Wildtieren offenbar überwiegend ebenfalls als Rohstoffe importiert - während des 1. Jt. v. Chr. echte Rara. Der Berberaffe erfuhr erst im weiteren Verlauf des 1. Jt. n. Chr. eine so starke Verbreitung, dass er auf Gibraltar heimisch wurde. ${ }^{141}$

Das archäologische Spektrum an Importgütern, sowie die Importmenge insgesamt überblickend sind die archäozoologischen Hinterlassenschaften eher spärlich. Der bei Pseudo-Skylax 112 erwähnte Handel der Phönizier mit Fellen, Häuten und anderen tierischen Produkten machte nur einen Bruchteil der im gesamtmediterranen Raum opportunistisch erworbenen und weitergegebenen Dinge aus. Beispielsweise bilden die auf dem italischen Festland entdeckten Reste von insgesamt 25 Straußeneiern aus der Spanne vom 7.-5. Jh. v. Chr. - als bei den Karthagern Straußeneier und daraus gefertigte Artefakte als Grabbeigaben besonders beliebt waren -weniger als $1 \%$

\footnotetext{
${ }^{140}$ Homig 2000, 177.

${ }^{141}$ Dazu Napier, Jenkins 1997, 122.
}

des überlieferten Importgutes. $^{142}$ Als Hauptimporte dieser Region zählte Fletcher Amulette vorwiegend ägyptischer Gottheiten (45\%) und mindestens 1173 Skarabäen (44\%).

Schriftliche und bildliche Quellen bezeugen für die griechische gleichermaßen wie die römische Kultur ein ausgeprägtes Interesse an fremden Geschöpfen. Dass, abgesehen von Elfenbein und Straußeneiern, Belege aus dem italischen Raum fehlen, lässt sich daher schwerlich anders als durch die lange und intensive Forschungsgeschichte erklären, im Verhältnis zu der die archäozoologische Tradition noch sehr jung ist. Eine größere Zahl an Nachweisen aus griechischen Tempelbezirken, wie ein besonderer Reichtum von Votivfunden im Allgemeinen, zeichnet dagegen die archaische Epoche (bis um 480 v. Chr.) aus. Kyrieleis stellt fest, wie diese Votive in späterer Zeit stärker durch eine andere Form der Göttergabe, etwa Geld, substituiert worden sind. ${ }^{143}$ Die Verwendung von Geld wird auch zunehmend den Handel geprägt haben, sodass Tiere und ihre Erzeugnisse nach und nach an Bedeutung als Zahlungsmittel verloren. Anderer wirtschaftlicher Nutzen und ein ideeller Wert dürften nun die primären Motivationen zum Transfer von Raritäten bilden.

\section{ZUSAMMENFASSUNG}

Betrachten wir die Erstnachweise von Tieren auf gesamteuropäischer Ebene, so ist zunächst festzustellen, dass während des 1. Jt. v. Chr. zu keiner Zeit die Verbreitung neuer Tiere vollständig stagnierte. Allerdings ist es die Etablierung von später typischen Haustieren, die sich im 10.-8. Jh. v. Chr. den europäischen Gestaden des Mittelmeeres fortsetzte - ein Prozess, der hier hauptsächlich im 2. Jt. v. Chr. eingesetzt hatte. Zwischen dem 8.-2. Jh. v. Chr. fanden ebenso Straußeneier Beliebtheit. Zur Etablierung von neuen Haustieren war deren Lebendtransport

\footnotetext{
${ }^{142}$ Fletcher 2007, 42 und 132.

${ }^{143}$ Kyrieleis 1988, 215.
} 
Voraussetzung. Anders präsentiert sich das Bild im Fall der Wildfauna. Häufig sind nur verschiedene Rohstoffe dieser Tiere belegt, selten ist davon auszugehen, dass ein Wildtier lebend über weite Strecken transportiert wurde. Transport und Haltung lebender Wildtiere waren je nach den Ansprüchen und dem Charakter der Tierart mit mehr oder weniger Schwierigkeiten verbunden. Die Funde des jung erwachsenen Berberaffen aus Nordirland und des Löwenjungen von Mogador fügen sich gut in die Beobachtung, dass die in jungem Alter bessere Anpassungsfähigkeit eine Zähmung und damit die Haltung des jeweiligen Tieres erleichtert.

Besonders die Bevölkerungen der Gebiete im Mittelmeerraum sind mit einer Vielzahl fremder Tiere mehr oder weniger vertraut gemacht worden. Die Gewöhnung an solche oftmals mit Luxus assoziierten, oder doch zumindest schwer zu beschaffenden, seltenen Güter schuf einen ständigen Bedarf an Nachschub, cen die Phönizier zu entwickeln und besonders die Griechen und später die Römer durch eigene Initiative $\mathrm{zu}$ pflegen wussten. Meiner Ansicht nach mündete dieses Phänomen zusammen mit anderen Faktoren in die seit hellenistischer Zeit spürbar wachsende Dekadenz insbesondere der Römer, die sich den Wert vieler seltener Tiere wörtlich durch den Magen gehen ließ und deren Interesse auch der weniger wohlhabenden Bevölkerungsschichten am Ausgefallenen durch Zurschaustellung exotischer Tiere und durch Tierkämpfe in den Arenen befriedigt wurde. Diese wertvollen, weil schwer zu beschaffenden Tiere können hier zweifelsohne zu den Prestigeobjekten zählen, die aus politischen Erwägungen heraus der mehr oder minder weit gefassten Öffentlichkeit zugänglich gemacht wurden - um Eindruck zu hinterlassen und die Gewogenheit der regelrecht Genusssüchtigen zu erhalten. Hauptsächlich die Schriftquellen und bisweilen künstlerische Quellen berichten von solchen Szenarien, die ihren Höhepunkt während der römischen Kaiserzeit erreichen sollten. Der archäozoologische Befund dagegen ist sehr dünn für die jüngere vorchristliche Zeit. U.a. diesem Phänomen sollte in zukünftigen Forschungen besonderes Augenmerk geschenkt werden. Zur Betrachtung gelangen müssen zu den hier vorgestellten Großsäugern und Vögeln auch die Kleinsäuger, Reptilien, Fische und Insekten etc., von denen im Rahmen dieses kurzen Artikels nur einzelne ausgewählte Beispiele angesprochen worden sind. Noch sind wir weit davon entfernt, das Bild der Importe exotischer Tiere im 1. Jt. v. Chr. in seiner Gesamtheit zu erfassen. Dazu gehört freilich auch die andere Seite der Medaille, nämlich Exporte von in Europa heimischen Tieren in die Gegenrichtung.

Bălășescu, Radu. 2004 A. Bălășescu, V. Radu, Omul şi animalele. Strategii si resurse la comunitătile Hamangia și Boian. Muz. Nat. Ist. a Romaniei. Biblioteca Muz. Nat. Ser. Cercetări Pluridisciplinare 9 (Tărgoviște 2004).

Banerjee, Rodrigues 1993 A. Banerjee, P. Rodrigues, Application of Infrared-Spectroscopy for the Identification of Gemstones in Archaeological Objects. Archäologisches Korrespondenzblatt,23, 1993, 251-258.

Banerjee, Schneider 1996 A. Banerjee, B. Schneider, Römisches Elfenbein - Zerstörungsfreie Materialprüfung mit optischen und spektralphotometrischen Methoden. Kölner Jahrbuch, 29, 1996, 331-342.

Barnett 1948

R.D. Barnett, Early Greek and Oriental Ivories. The Journal of Hellenic Studies, 68, 1948, 1-25.

Bell 1988

M. Bell, Excavations at Morgantina, 1980-1985. Preliminary Report XII. American Journal of Archaeology, 92,3, 1988, 313-342. 


\section{Benecke 1994}

\section{Boessneck 1953}

\section{Boessneck 1988}

Bodson 1998

\section{Bodson 2005}

Bökönyi 1983

Boessneck, von den Driesch 1981

Boessneck, von den Driesch 1983

Boessneck, von den Driesch 1988

\section{Borell 1978}

\section{Brentjes 1965}

Caubet 2000

\section{Clutton-Brock 1999}

Cucchi, Vigne, Auffray 2005

\section{Davis, MacKinnon 2009}

De Angelis 2001

Dennis 1848

Deshours 2004

Dorst, Dandelot 1973

Edwards 1998

\section{Edwards et alii 2006}

Norbert Benecke, Der Mensch und seine Haustiere. Die Geschichte einer jahrtausendealten Beziehung (Stuttgart 1994).

Joachim Boessneck, Die Haustiere in Altägypten. Veröff. Zool. Staatsslg. München 3 (München 1953).

Joachim Boessneck, Die Tierwelt des Alten Ägypten untersucht anhand kulturgeschichtlicher und zoologischer Quellen (München 1988).

L. Bodson, Contribution à l'étude des critères d'appréciation de l'animal exotique dans la tradition grecque ancienne. In: L. Bodson ( $\mathrm{Hg}$.), Les animaux exotiques dans les relations internationals: espeèces, functions, significations. Journée d'étude Université de Liège, 22 mars 1997. Coll. hist. connaissances zoologiques 9 (Liège 1998) 139-212.

L. Bodson, Naming the exotic animals in ancient Greek and Latin. In: A. Minelli, G. Ortelli, G. Sanga (Hg.), Animal Names (Venezia 2005) 453-480.

S. Bökönyi, Animal bones from test excavations of early Neolithic ditched villages on the Tavoliere, South Italy. In: S.M. Cassano, A. Manfredini (Hrsg.), Studi sul Neolitico del Tavoliere della Puglia. BARIntSer 160 (Oxford 1983) 237-249.

J. Boessneck, A. von den Driesch, Reste exotischer Tiere aus dem Heraion auf Samos. $A M 96$ (Berlin 1981) 245-248.

J. Boessneck, A. von den Driesch, Weitere Reste exotischer Tiere aus dem Heraion auf Samos. $A M 98$ (Berlin 1983) 21-24.

J. Boessneck, A. von den Driesch, Knochenabfall von Opfermahlen und Weihgaben aus dem Heraion von Samos (7. Jh. v. Chr.) (München 1988).

B. Borell, Attisch geometrische Schalen. Eine spätgeometrische Keramikgattung und ihre Beziehungen zum Orient. Keramikforschungen 2 (Mainz a. R. 1978).

B. Brentjes, Der geschichtliche Tierwechsel in Vorderasien und Nordafrika in altertumskundlicher Sicht. Säugetierkundliche Mitteillungen 13,3, 1965, 101-109.

A. Caubet, Egyptien ou egyptisant? L'egyptomanie est vieille de cinq mille ans. In: J.-C. Béal, J.-C. Goyon (Hrsg.), Des Ivories et des Cornes dans les mondes anciens (orient - occident). Collect. Inst. Archéologie et Hist. Ant. Univ. Lumière-Lyon 2,4 (Paris 2000) 119-123.

J. Clutton-Brock, Natural History of Domesticated Mammals, Cambridge $1999^{2}$.

T. Cucchi, J.-D. Vigne, J.-C. Auffray, First occurrence of the house mouse (Mus musculus Domesticus Schwarz \& Schwarz, 1943) in the Western Mediterranean: a zooarchaeological revision of subfossil Occurrences. Biological Journal of the Linnean Society 84, 2005, 429-445.

S. Davis, M. MacKinnon, Did the Romans bring fallow deer to Portugal? Environmental Arch. 14, 1, 2009, 15-26.

F. de Angelis, Archaeology in Sicily 1996-2000. Archaeological Reports, 47, $2000,2001,145-201$.

G. Dennis, The cities and cemeteries of Etruria I (London 1848).

$\mathrm{N}$. Deshours, Les institutions civiques de Messène à l'époque hellénistique tardive. Zeitschrift für Papyrologie und Epigraphik, 150, 2004, 134-146.

J. Dorst, P. Dandelot, Säugetiere Afrikas. Ein Taschenbuch für Zoologen und Naturfreunde (Hamburg, Berlin 1973).

H.G.M. Edwards, D.W. Farwell, J.M. Holder, E.E. Lawson, Fourier transform Raman spectroscopy of ivory: a non-destructive diagnostic technique. Studies in Conservation, 43, 1998, 9-16.

H.G.M. Edwards, Rachel H. Brody, N.F.N. Hassan, D.W. Farwell, S. O'Connor, Identification of archaeological ivories using FT-Raman spectroscopy. Analytica Chimica Acta 559, 2006, 64-72. 
Felsch 1987

Fletcher 2007

Friedman 2003

Friedman 2004

Furtwängler 1980

Gambart, Podvin 2009

Guggisberg 1972

Haas 1953

Herre, Röhrs 1990

Isserlin,

Du Plat Taylor 1974

Jeitteles 1872

Haimovici 2007

Hornig 2000

Hünemörder 2004

Keller 1909

King 1953

Kopcke 1968

Krzyszkowska 1990

Kyrieleis 1988

Lafrenz 2003
R.S.C. Felsch, Kalapodi. Bericht über die Grabungen im Heiligtum der Artemis Elaphebolos und des Apollon von Hyampolis 1978-1982, Arch. Anz. 1987, 1-26.

R.N. Fletcher, Patterns of Imports in Iron Age Italy. BARIntSer 1732 (Oxford 2007).

R.F. Friedman, Excavating an Elephant. Nekhen News 15, 2003, 9-10.

R.F. Friedman, Elephants at Hierakonpolis. In: S. Hendrickx, R.F. Friedman, K.M. Ciałowicz, M. Chłodnicki (Hrsg.), Egypt at its Origins. Studies in Memory of Barbara Adams. Proceedings of the International Conference "Origin of the State. Predynastic and Early Dynastic Egypt", Krakow, 28th August-1st September 2002. OLA 138 (Leuven 2004) 131-168.

A.E. Furtwängler, Heraion von Samos: Grabungen im Südtemenos 1977, I. Schicht- und Baubefund, Keramik. $A M 95,1980,149-224$.

P. Gambart, J.-L. Podvin, Le crocodil sur les parois de tombes de l'Ancien et du Moyen Empire. GöttMisz 221, 2009, 29-36.

C.A.W. Guggisberg, Crocodiles. Their Natural History, Folklore and Conservation (Trowbridge, London 1972).

G. Haas, On the Occurrence of Hippopotamus in the Iron Age of the Coastal Area of Israel (Tell Qasîleh). Bulletin of the American Schools of Oriental Research, 132, 1953, 30-34.

W. Herre, M. Röhrs, Haustiere - zoologisch gesehen (Stuttgart, New York $\left.1990^{2}\right)$.

B.S.J. Isserlin, J. Du Plat Taylor, Motya. A Phoenician and Carthaginian city in Sicily 1. Fieldwork and excavation (Leiden 1974).

L.H. Jeitteles, Die vorgeschichtlichen Alterthümer der Stadt Olmütz und ihrer Umgebung. Mitteilungen der Anthropologischen Gesellschaft in Wien, 2, 1872, 18-32; 53-62; 86-90; 130-136; 162-182; 211-224; 233-247; 278-285.

S. Haimovici, Mediterranean species discovered among the animal remains from Dobrodgea province, neolithic to eneolithic period. Analele Stiin. Univ. "Al. I. Cuza" Iaşi N. S. Sec. Iia Biologie animala 53, 2007, 291-302.

K. Homig, Großtiertransporte nach und innerhalb Europas in der Antike methodische Probleme, Fallbeispiele und kulturelle Rezeption. In: F. Lüth, U. Schoknecht (Hrsg.), Schutz des Kulturerbes unter Wasser. Veränderungen europäischer Lebenskultur durch Fluß- und Seehandel. Beiträge zum Internationalen Kongreß für Unterwasserarchäologie (IKUWA '99) 18.-21. Februar 1999 in Sassnitz auf Rügen. Beitr. Ur- u. Frühgesch. MecklenburgVorpommerns 35 (Lübstorf 2000) 177-185.

Brill's New Pauly, Encyclopedia of the Ancient World V, 2004, 716-717 s.v. Gazelle (Christian Hünemörder).

O. Keller, Die antike Tierwelt 1. Säugetiere (Leipzig 1909).

J.E. King, Mammal bones vom Khirokita and Erimi. In: P. Dikaios (Hg.), Khirokita. Final report on the excavation of a Neolithic settlement in Cyprus on behalf of the Department of Antiquities, 1936-1946. App. 111 (Oxford 1953) 431-437.

G. Kopcke, Heraion von Samos. Die Kampagnen 1961, 1965 im Südtemenos (8.-6. Jahrhundert). $A M$ 83, 1968, 240-314.

O. H. Krzyszkowska, Ivory and Related Materials: An Illustrated Guide. Classical Handbook 3, Bull. Suppl. 59 (London 1990).

H. Kyrieleis, Offerings of 'the Common Man' in the Heraion at Samos. In: R. Hägg, N. Marinatos, G.C. Nordquist (Hrsg.), Early Greek Cult Practice. Proceedings of the Fifth International Symposium at the Swedish Institute at Athens, 26-29 June, 1986. Skrifter Utgivna Svenska institutet i Athen 4, 38 (Stockholm 1988) 215-221.

K.A. Lafrenz, Tracing the Source of the Elephant and Hippopotamus Ivory from the 14th Century B.C. Uluburun Shipwreck: The Archaeological, Historical, and Isotopic Evidence (Unpubl. Masterarbeit South Florida 2003). 


\section{Lenz 1951}

Lipiński 2004

Lynn 1997a

Lynn 1997b

Mallory 1997

Marzoli, El Khayari 2009 D. Marzoli, A. El Khayari, Mogador (Essaouira, Marokko). Vorbericht über

die Kampagnen 2006 und 2007. Madrider Mitteilungen, 50, 2009, 80-101.

Mayor 2000

Mayor 2001

Matthews 2002

Meuli 1946

Müth 2007

Napier, Jenkins 1997

Ninov 1999

Nobis 2001

\section{Ostby et alii 1994}

Pascal, Lorvelec, Vigne 2003

\section{Peters,}

von den Driesch 1990

\section{Phillips 2008}

C. Lenz, Vergleichende Betrachtungen an Antilopen. Die Systematik der Gattungen Alcelaphus und Strepsiceros (Diss. Kiel 1951).

E. Lipiński, Itineraria Phoenicia. Orientalia Lovaniensia analecta 127. Stud. Phoenicia 18 (Leuven 2004).

C.J. Lynn, The date of the deposition of the ape cranium. In: D. M. Waterman, Excavations at Navan Fort 1961-71. Northern Ireland Arch. Monogr. 3 (Belfast 1997) 125.

C.J. Lynn, Comparisons and interpretations. In: D.M. Waterman, Excavations at Navan Fort 1961-71. Northern Ireland Arch. Monogr. 3 (Belfast 1997) 209-230.

J.P. Mallory, Emain Macha and Navan Fort. In: D.M. Waterman, Excavations at Navan Fort 1961-71. Northern Ireland Arch. Monogr. 3 (Belfast1997) 197-207. A. Mayor, A Time of Giants and Monsters. The discovery of huge bones in antiquity spawned vivid and imaginative myths. Archaeology 53,2, 2000, 58-61.

A. Mayor, The First Fossil Hunters. Paleontology in Greek and Roman Times (Princeton 2001).

R. Matthews, Zebu: harbingers of doom in Bronze Age westem Asia? Antiquity. A Quarterly Review of Archaeology, 76, 2002, 438-446.

K. Meuli, Griechische Opferbräuche. In: O. Gigon, K. Meuli, W. Theiler, F. Wehrli, B. Wyss (Hrsg.), Phyllobolia. Für Peter von der Mühll zum 60. Geburtstag am 1. August 1945 (Basel 1946) 185-288.

S. Müth, Eigene Wege: Topographie und Stadtplan von Messene in spätklassisch-hellenistischer Zeit. Internationale Archäologie 99 (Rahden, Westf. 2007).

P.H. Napier, P.D. Jenkins, Monkey skull and mandible of the Iron Age found at site B. In: D.M. Waterman, Excavations at Navan Fort 1961-71. Northern Ireland Arch. Monogr. 3 (Belfast 1997) 120-124.

L.K. Ninov, Vergleichende Untersuchungen zur Jagd und zum Jagdwild während des Neolithikums und Äneolithikums in Bulgarien. In: N. Benecke (Hrsg.), The Holocene History of European Vertebrate Fauna. Modern Aspects of Research. Workshop, 6th to 9th April 1998, Berlin. Archäologie in Eurasien 6 (Rahden/Westf. 1999) 323-338.

G. Nobis, Archäozoologische Studien an Tierresten aus Alt-Messene/Ithome (SW-Peloponnes, Griechenland), Grabungen 1992 bis 1996. In: H. Buitenhuis, W. Prummel (Hrsg.), Animals and Man in the Past. Essays in honor of Dr. A.T. Clason emeritus professor of archaeozoology Rijksuniversiteit Groningen, the Netherlands. ARC-Publicatie 41 (Groningen 2001) 95-121.

E. Ostby, J.-M. Luce, G.C. Nordquist, C. Tarditi, M.E. Voyatzis, The sanctuary of Athena Alea at Tegea: first preliminary report (1990-1992). Opuscula Atheniensia, 20,8, 1994, 89-140.

M. Pascal, O. Lorvelec, J.-D. Vigne, Le Daim européen: Dama dama (Linné, 1758). In: M. Pascal, O. Lorvelec, J.-D. Vigne, P. Keith, Ph. Clergeau (Hrsg.), Évolution holocène de la faune de Vertébrés de France: Invasions et disparitions (Paris 2003) 296-297.

J. Peters, A. von den Driesch, Archäozoologische Untersuchung der Tierreste aus der kupferzeitlichen Siedlung von Los Millares (Prov. Almería). In: A. von den Driesch (Hrsg.), Neolithische und Kupferzeitliche Tierknochenfunde aus Südspanien. Los Castillejos. Los Millares. Studien über frühe Tierknochenfunde von der Iberischen Halbinsel 12 (München 1990) 51-115.

J.S. Phillips, Aegyptiaca on the island of Crete in their chronological context: A critical review 1-2. Contributions to the Chronology of the Eastern Mediterranean 18 (Vienna 2008). 


\section{Poplin 1993}

\section{Poplin 1994}

Poplin 1996

Reese 2000

\section{Ringe 1959}

Ryder 1975

Sakellarakis 1990

Schmidt 1919

Schüle 1993

Shimoyama et alii 1998

Shimoyama, Morimoto, Ozaki 2004

\section{Stanzel 1991}

Sykes 2004

Thenius, Vávra 1996

Trantalidou 2000
F. Poplin, Que l'homme cultive aussi bien le sauvage que le domestique. In: J. Desse, F. Audoin-Rouzeau (Hrsg.), Exploitation des animaux sauvages a travers le temps. IV colloque international de l'homme et l'animal. Société de recherche interdisciplinaire. Actes des rencontres $15-16-17$ octobre 1992 (Juan-les-Pins 1993) 527-539.

F. Poplin, Menschen- und Pferdeknochen in Viereckschanzen am Beispiel von Gournay-sur-Aronde (Nordfrankreich). In: M. Kokabi, J. Wahl (Hrsg.), Beiträge zur Archäozoologie und Prähistorischen Anthropologie. 8. Arbeitstreffen der Osteologen Konstanz 1993 im Andenken an Joachim Boessneck. FBerBadWürt 53 (Stuttgart 1994) 315-322.

F. Poplin, Les cerfs harnachés de Nogent-sur-Seine et le statut du cerf antique. $C R A I 140,1,1996,393-421$.

D.S. Reese, Ostrich eggs. In: J.W. Hayes, A.S. Walker, M.K. Dabney, N.J. Skon-Jedele, J.W. Shaw, D.K. Harlan, K.A. Schwab, D.S. Reese, P.J. Anderson, Miscellaneous Finds. In: J.W. Shaw, M.C. Shaw (Hrsg.), Kommos IV. The Greek Sanctuary I (Princeton 2000) 401-403.

D. Ringe, Über das autochthone nacheiszeitliche Vorkommen des Damhirsches - Cervus (Dama) dama L. - in Europa (Diss. München 1959).

M.L. Ryder, Some Phoenician animal remains from Sicily. In: A.T. Clason (Hrsg.), Archaeozoological studies. Papers of the Archaeozoological Conference 1974, held at the Biologisch-Archaeologisch Instituut of the State University of Groningen (Amsterdam, Oxford 1975) 213-218.

J.A. Sakellarakis, The Fashioning of Ostrich-Egg Rhyta in the CretoMycenaean Aegean. In: D.A. Hardy, Chr.G. Doumas, J.A. Sakellarakis, P.M. Warren (Hrsg.), Thera and the Aegean World 3,1. Archaeology. Proceedings of the Third International Congress, Santorini, Greece, 3-9 September 1989 (London 1990) 285-308.

K.P. Schmidt, Contributions to the herpetology of the Belgian Congo based on the collection of the American Museum Congo Expedition, 1909-1915 1. Turtles, crocodiles, lizards, and chameleons. Bulletin of the American Museum of Natural History 39,20, 1919, 385-624.

W. Schüle, Mammals, vegetation and the initial human settlements of the Mediterranean islands: a palaeoecological approach. Journal of Biogeography 20, 1993, 399-412.

M. Shimoyama, T. Nakanishi, Y. Hamanaga, T. Ninomiya, Y. Ozaki, Nondestructive discrimination between elephant ivory products and mammoth tusk products by glancing incidence $\mathrm{X}$-ray fluorescence spectroscopy. Journal of Trace and Microprobe Techniques 16, 1998, 175-182.

M. Shimoyama, S. Morimoto, Y. Ozaki, Non-destructive analysis of the two subspecies of African elephants, mammoth, hippopotamus, and sperm whale ivories by visible and short-wave near infrared spectroscopy and chemometrics. The Analyst 129, 2004, 559-563.

M. Stanzel, Die Tierreste aus dem Artemis-, Apollon-Heiligtum bei Kalapodi in Böotien, Griechenland (Diss. München 1991).

N. Sykes, The Introduction of Fallow Deer to Britain: A Zooarchaeological Perspective. Environmental Arch. 9,1, 2004, 75-84.

E. Thenius, N. Vávra, Fossilien im Volksglauben und im Alltag (Frankfurt a.M. 1996).

K.T Trantalidou, Animal Bones and Animal Representations at Late Bronze Age Akrotiri. In: S. Sherratt (Hrsg.), The Wall Paintings of Thera. Proceedings of the First International Symposium 2. Proceedings of the First International Symposium, Petros M. Nomikos Conference Center, Thera, Hellas. 30 August-4 September 1997 (Athen 2000) 709-735.

\section{Trutnau 1994}

L. Trutnau, Krokodile. Alligatoren, Kaimane, Echte Krokodile und Gaviale. Neue Brehm-Bücherei 593 (Magdeburg 1994). 
Uerpmann 1971

Uerpmann 1987

Uerpmann, Uerpmann 1986

\section{Villari 1986}

Von Berneck 1875

Von Koenigswald 2002

Warner 1997a

Warner 1997b

Waterman 1997

Yannouli, Trantalidou 1999
H.-P. Uerpmann, Die Tierknochenfunde aus der Talayot-Siedlung von S'Illot (San Lorenzo, Mallorca). Studien über frühe Tierknochenfunde von der Iberischen Halbinsel 2 (München 1971).

H.-P. Uerpmann, The ancient distribution of ungulate mammals in the Middle East. Fauna and archaeological sites in Southwest Asia and Northeast Africa. Beih. TAVO A 27 (Wiesbaden 1987).

H.-P. Uerpmann, M. Uerpmann, Tierknochenfunde aus der phönizischen Faktorei von Toscanos und anderen phönizisch beeinflussten Fundorten der Provinz Málaga in Südspanien. Studien über frühe Tierknochenfunde von der Iberischen Halbinsel 4 (München 1973) 35-100.

P. Villari, Nota preliminare allo studio della faune della tarda preistoria della Sicilia Orientale. Studia Ecologia Quaternario 8, 1986, 169-76.

K.G. von Berneck, Bilder-Atlas. Anatomie, Zoologie, Botanik, Mineralogie. Ikonographische Encyklopaedie der Wissenschaften und Kuenste. Ein Ergänzungswerk zu jedem Conversations-Lexikon 2. Zoologie (Leipzig 1875).

W. von Koenigswald, Lebendige Eiszeit. Klima und Tierwelt im Wandel (Darmstadt 2002).

R.B. Warner, The non-ceramic artefacts and the chronology of site B. In: D.M. Waterman, Excavations at Navan Fort 1961-71. Northern Ireland Arch. Monogr. 3 (Belfast 1997) 100-105.

R.B. Warner, The radiocarbon chronology of the Navan excavations. In: D.M. Waterman, Excavations at Navan Fort 1961-71. Northern Ireland Arch. Monogr. 3 (Belfast 1997) 173-196.

D.M. Waterman, Excavations at Navan Fort 1961-71. Northern Ireland Arch. Monogr. 3 (Belfast 1997)

E. Yannouli, K. Trantalidou, The fallow deer (Dama dama Linnaeus, 1758): Archaeological presence and representation in Greece. In: N. Benecke (Hrsg.), The Holocene History of European Vertebrate Fauna. Modern Aspects of Research. Workshop, 6th to 9th April 1998, Berlin. Archäologie in Eurasien 6 (Rahden, Westf. 1999) 247-281.

\section{LIST OF PLATE}

1. Heraion of Samos. Right homcore of a North African hartebeest Alcelaphus b. b. (7th century BC) in lateral (left) and frontal view (right) (Drawing M. Mahn after Boessneck, von den Driesch 1981, pl. 87,2-3). 2. North African hartebeest Alcelaphus buselaphus (M. Mahn).

3. Nile crocodile Crocodylus niloticus. Scull from palatal (left) and dorsal view (right) (after Schmidt 1919, 422-423, fig. 3-4).

4. Sanctuary of Kalapodi, Phokis (Greece). Burnt fragment of a Dorcas gazelle Gazella dorcas homcore (690-480 BC) (Drawing after Stanzel 1991, pl. 1,3).

5. Dorcas gazelle Gazella dorcas (M. Mahn).

6. Dama gazelle Nanger dama (M. Mahn).

7. Barbary ape Macaca sylvana (after von Berneck 1875, pl. 1,8).

8. Navan Fort, County Armagh (Northern Ireland). Cranium of a five to six years old male Barbary ape (220-95 BC) (Drawing M. Mahn after Waterman 1997, pl. 28).

Mirjam MAHN, M.A.

Brandvorwerkstr. 77

04275 Leipzig

mirjam.mahn@web.de 


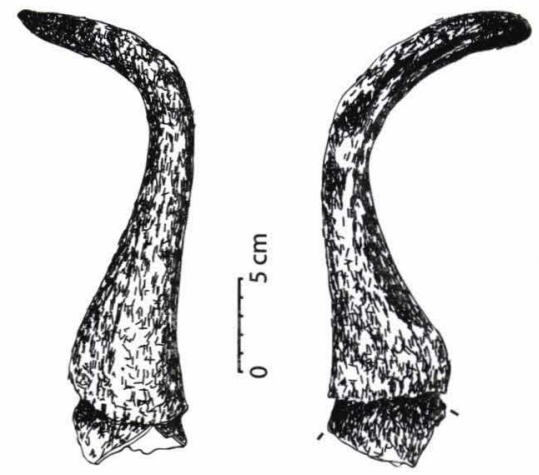

Abb. 1. Heraion von Samos. Rechter Hornzapfen der Nordafrikanischen Kuhantilope Alcelaphus buselaphus buselaphus (7. Jh. V. Chr.) in Lateral - (links) und Frontalanisicht (rechts). M. Mahn apud Boessneck, von den Driesch 1981, pl. 97, 2-3.
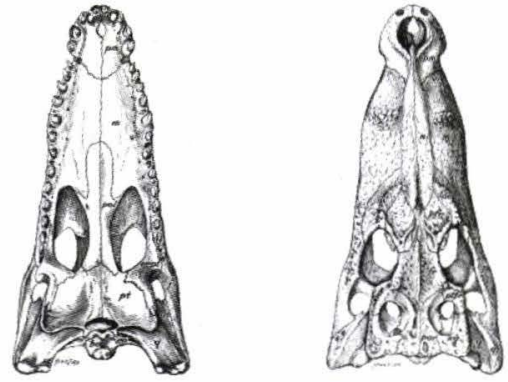

Abb. 3. Nilkrokodil Crocodylus niloticus. Schädel von palatal (links) und dorsal (rechts). Apud Schmidt 1919, 422-423, fig. 3-4.

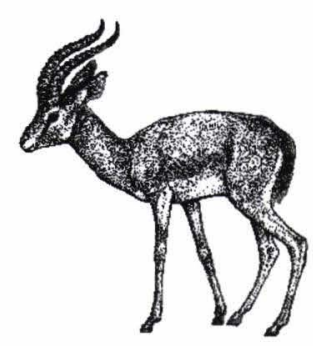

Abb. 5. Dorkasgazelle Gazella dorcas (M. Mahn).

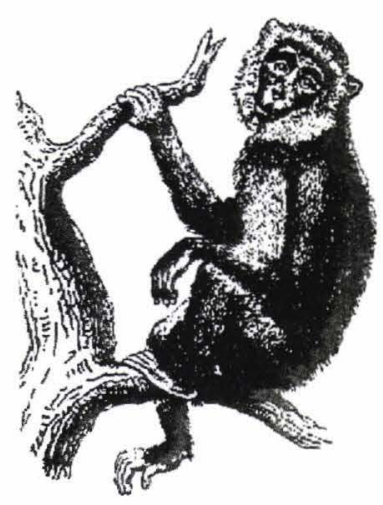

Abb. 7. Berberaffe Macaca sylvana. Apud von Berneck 1975, pl. 1, 8 .

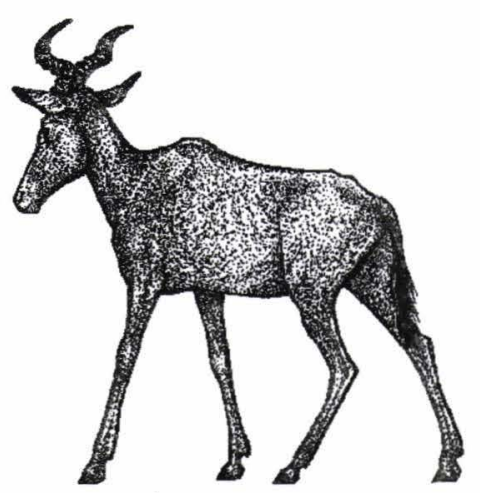

Abb. 2. Kuhantilope Alcelaphus buselaphus (M. Mahn).
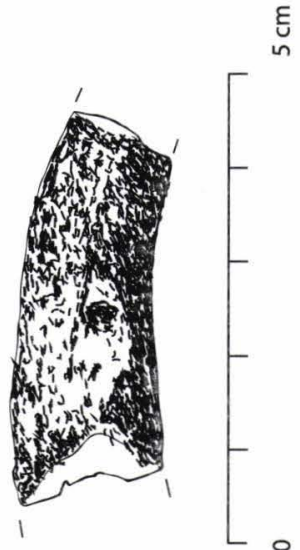

Abb. 4. Heiligtum von Kalapodi, Phokis (Griechenland). Verbranntes Hornzapfenfragment der Dorkasgazelle Gazella dorcas (690-480 v. Chr.). Apud Stanzel 1991, pl. 1, 3.



Abb. 6. Damagazelle Nanger dama (M. Mahn).

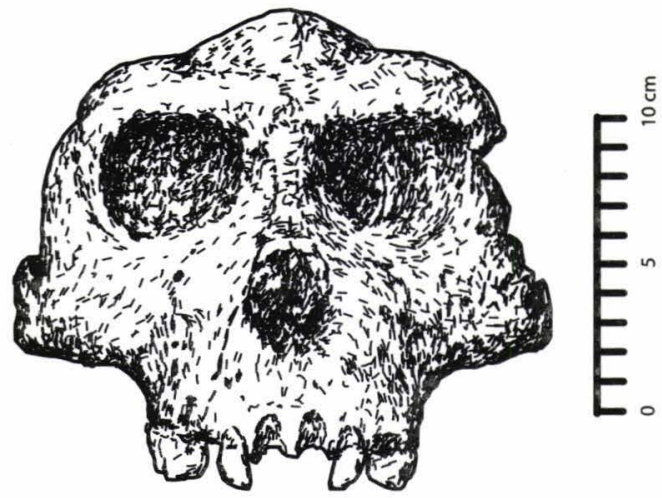

Abb. 8. Navan Fort, County Armagh (Nordirland). Cranium eines fünf - bis sechsjährigen, männlichen Berberaffen (220-95 v. Chr.). M. Mahn apud Waterman 1997, pl. 28. 